\title{
Screening for Social Determinants of Health in Pediatric Resident Continuity Clinic
}

$$
\text { By }
$$

\author{
Marcia A. Morgenlander MD
}

A Master's Paper submitted to the faculty of the University of North Carolina at Chapel Hill in partial fulfillment of the requirements for the degree of Master of Public Health in the Public

Health Leadership Program

Chapel Hill 


\begin{abstract}
BACKGROUND: The American Academy of Pediatrics and the Academic Pediatric Association have published guidelines supporting screening and referral for social determinants of health $(\mathrm{SDH})$ in pediatric primary care. Despite this charge, little is known about the current prevalence of screening practices taking place in medical homes across the country.
\end{abstract}

OBJECTIVE: The objective of this study is to describe current practices for systematically screening for social determinants of health in nationwide pediatric continuity clinics enrolled in the CORNET network.

METHODS: We recruited 144 pediatric resident continuity clinics enrolled in the Continuity Research Network (CORNET) of the Academic Pediatric Association. Continuity clinic directors at 75 sites agreeing to participate received an electronic survey instrument requesting clinic demographics and information on screening and educational practices for fifteen social determinants of health.

RESULTS: In the sixty-four clinic sites that responded to the survey, the range of SDH being screened for was 0-15 with a mean of 7 . The most commonly screened SDH were maternal depression (86\%), child education (84\%), food insecurity (71\%) and firearm exposure (65\%). Most commonly, screening instruments are paper documents original to the clinic. Primary 
providers, nurses or medical assistants administer the majority of screens on rooming or during the encounter. Clinics not currently screening have plans to begin screening for nearly a quarter of those SDH not currently being screened within the next 3 years. Clinic directors most often cited lack of time (63\%), lack of resources to address positive screen (50\%) and inadequate training (46\%) as barriers to SDH screening. Less than $10 \%$ of resident continuity clinic directors cited lack of indication or evidence for screening as barriers.

CONCLUSIONS: Screening for SDH in pediatric resident continuity clinics has not yet been universally implemented. Screening practices are variable reflecting the complex nature of screening and the heterogeneity of the various SDH. Characteristics of commonly screened SDH include validated, concise screening tools, longstanding Bright Futures recommendations, literature suggesting benefit, and identifiable interventions. The major barriers to SDH screening are lack of time and lack of resources to address positive screens. A variety of measures including comprehensive, concise screening tools, up-to-date community resource guides, streamlined referral processes, embedded multidisciplinary teams, and strong community partnerships could mitigate these barriers. 


\section{ACKNOWLEDGEMENTS}

I would first and foremost like to thank Dr. Samantha Schilling, my research mentor and second reader, for freely providing her time, patience and guidance in the development and execution of this study. Next, I would like to thank Dr. Mike Steiner for welcoming me into the Department of Pediatrics when I arrived at UNC as a Preventive Medicine Resident and for introducing me to Dr. Schilling. In addition to Drs. Schilling and Steiner, I would like to thank the other members of our research team, Dr. Janet Serwint, and Dr. Lynn Garfunkel. I would also like to acknowledge the expertise and invaluable contributions of Clarence Potter and Teresa Edwards to the development of our survey instrument. This study was only possible through the support of the CORNET Research Network of the APA, and specifically through the work of Ms. Hollyce Tyrrell, Research and Network Coordinator who was the recruitment point of contact for all CORNET institutions. Finally, I would like to thank Dr. Sue Tolleson-Rinehart, my academic advisor and first reader, for her tremendous effort and contribution to the completion of this Master's Paper. 


\section{TABLE OF CONTENTS}

$\begin{array}{ll}\text { Title Page } & \text { i }\end{array}$

$\begin{array}{lll}\text { Abstract } & \text { ii }\end{array}$

$\begin{array}{ll}\text { Acknowledgements } & \text { iii }\end{array}$

Table of Contents $\quad$ iv

List of Tables $\quad$ V

List of Figures $\quad$ vi

$\begin{array}{ll}\text { Introduction } & 1\end{array}$

Methods 2

$\begin{array}{ll}\text { Results } & 4\end{array}$

$\begin{array}{ll}\text { Discussion } & 6\end{array}$

$\begin{array}{ll}\text { Limitations } & 14\end{array}$

$\begin{array}{ll}\text { Conclusion } & 14\end{array}$

$\begin{array}{ll}\text { References } & 16\end{array}$

$\begin{array}{ll}\text { Figure } & 19\end{array}$

$\begin{array}{ll}\text { Tables } & 20\end{array}$

Appendix A: Survey Instrument 30

Appendix B: Limited Systematic Review 


\section{LIST OF TABLES}

Table 1 - Demographics of Participating Clinics

Table 2 - Summary of Social Determinant Screening

Table 3 - Source of Screening Tool for each SDH

Table 4 - Visit Type When SDH Screening Occurs

Table 5 - Method of Screening Administration

Table 6-Staff Member Administering Screening

Table 7 - Timing of SDH Screening

Table 8 - Staff Member Following Up Screening

Table 9 - Plan to Screen in Next 3 Years

Table 10 - Barriers to SDH Screening 


\section{LIST OF FIGURES}

Figure 1 - Clinics Screening for Each SDH 


\section{Introduction}

Social determinants of health (SDH) are the economic, environmental and family contextual factors which integrate with biology, genetics, and behavior to produce health outcomes. Unfavorable social determinants are root causes of poor health in childhood and across the lifespan. ${ }^{1-4}$

Social determinants of health may worsen outcomes directly as when mold exposure or passive smoke exacerbate asthma. ${ }^{5,6}$ Less directly, early adversity from exposure to violence, food insecurity, housing instability or parental dysfunction, works through the common pathway of toxic stress to compromise physical health, socio-emotional development and educational achievement. $^{7-9}$ Stress disrupts neuroendocrine, inflammatory and immune system functioning leading to illnesses including depression, suicide, substance abuse, adult obesity, heart disease and cancer. ${ }^{7-12}$

Children living in poverty are particularly vulnerable. ${ }^{1} 20 \%$ of US children live in poverty. ${ }^{13}$ Half of these children will read below grade level in $4^{\text {th }}$ grade. ${ }^{14}$ Each of these children is five times as likely to be maltreated as those with financial stability. ${ }^{15}$ In addition to improving health for the one in five children living in poverty, targeting SDH early in life could potentially improve health outcomes for the two in three US adults who report exposure to childhood adversity. ${ }^{9}$

For these reasons, pediatricians are increasingly motivated to work outside of the traditional biomedical model and to address social determinants of health in practice. ${ }^{15-17}$ In 2013 , both the American Academy of Pediatrics (AAP) and the Academic Pediatric Association (APA) created task forces on child poverty. ${ }^{14,18}$ As a result, in 2016 the American Academy of Pediatrics 
(AAP) published a policy statement describing the effects of poverty on children and calling on pediatric health practitioners in family centered medical homes to assess the financial stability of families, link families to resources and coordinate care with community partners. ${ }^{19}$ An APA work group also published a 2016 guideline for clinicians highlighting the importance of addressing SDH and outlining screening tools and resources to do so. ${ }^{14}$

Despite the importance of SDH measurement, few pediatric primary care providers have routinely incorporated such screening into their practics. ${ }^{20}$ Even amidst the growing consensus that medical homes have the potential to help mitigate the effect of adverse social determinants on children's health, little is known about current screening practices taking place across the country. We hypothesize that levels of screening for social determinants of health in pediatric practice remain below the universal level recommended by both the AAP and the APA. This study will examine and inform current practice on screening for SDH in pediatric resident continuity clinics.

\section{Methods}

\section{Study Design and Oversight}

This cross-sectional, observational study used a REDCap electronic survey instrument to collect data from pediatric resident continuity clinic directors who are members of the Continuity Research Network of the Academic Pediatric Association, CORNET. CORNET members collaborate to study residency education and practice with the goals of improving health care delivery to underserved children and increasing residency participation in primary care

research. ${ }^{21}$ The study was approved by the CORNET executive committee in October of 2016. 
The study protocol was reviewed by the Institutional Review Board of the Office of Human Research Ethics of the University of North Carolina (UNC) and was determined to be Not Human Subjects Research.

The REDCap survey instrument was designed in consultation with the UNC REDCap bioinformatics team and the UNC Odum Institute for Social Science Research. The survey consisted of three main sections and requested data from academic year July 1, 2015 through June 30, 2016. The first section requested demographic information for each clinic site and its resident and patient populations. It also asked about the presence of multidisciplinary team members such as social workers. The second section asked about resident education practices. The final section queried patient screening practices for each SDH. The 2016 American Academy of Pediatrics guidelines provided an overview of the potential harms attributable to among children living in poverty, and described practical screening tools and resources for clinicians. ${ }^{22}$ We collected data on SDH domains included in these guidelines: child maltreatment, family financial support, intimate partner violence, maternal depression/family mental illness, household substance abuse, parental health literacy, parental stress, childcare needs, child education, physical environment (home and neighborhood), food insecurity, parental incarceration, immigration, and firearm exposure.

For each SDH, we asked whether residents were receiving didactic or skills training and whether the practice had systematic screening in place. If screening was in place, we requested logistical details. If screening was not occurring, we asked about plans to implement it within 3 years and about perceived barriers. The complete survey instrument can be found in Appendix A. 


\section{Enrollment and Procedures}

Study enrollment took place from April to November, 2017. Following construction and piloting of the survey instrument, we obtained continuity clinic site information from CORNET. Each of the 144 individual continuity clinic sites of the 115 member institutions of CORNET was eligible for enrollment. Member institutions received an invitation letter to the approved study via the CORNET list serve. Interested institutions contacted the CORNET research coordinator and provided contact information for continuity clinic sites and directors. Once an institution agreed to participate, the CORNET research coordinator compiled contact information for its continuity clinic sites and released it to the research team on a weekly basis. Through REDCap, a unique survey link was emailed to each continuity clinic site director. Three weekly automated reminders, and at least one personal email followed until the survey instrument was completed or until data collection closed. In June, 2017, CORNET regional research chairs emailed their institutions to encourage participation. Participants received a $\$ 25$ gift card for survey submission.

\section{Results}

Seventy-five of the 144 CORNET sites (52\%) agreed to participate in the study and received a REDCap survey link. Sixty-four of those 75 programs (85\%) returned surveys, for a final response rate by October 27, 2017 of 64/144 (44\%) continuity clinic sites, and 47 of the 115 member institutions (41\%).

Demographics of participating clinics can be seen in Table 1. More responding clinics were located in the South (43.8\%) than in the other three regions of the United States. Patients in these clinics were predominantly racial minorities (65\%) and insured through Medicaid (72\%). Most clinics embedded a social worker (83\%). 
Table 1 about here.

Our survey asked each clinic site about screening practices for fifteen discrete SDH. The mean number of SDH being screened for was 7. The range was 0-15 with 2 clinics reporting no screening, and 2 clinics screening for all 15 SDH. As shown in Figure 1, maternal depression/family mental illness was screened for in $86 \%$ of clinics, making it the most commonly screened SDH. Next in screening frequency were child education (84\%), food insecurity $(71 \%)$ and firearm exposure (65\%). Clinics most infrequently screened for immigration status (17\%), parental health literacy (19\%) and parental incarceration $(21 \%)$. See Table 2 for further detail about the prevalence of screening.

Figure 1 about here

Table 2 about here

When screening does occur, screening instruments are most likely to be original to the clinic (45.7\%), rather than from a published or other external source, and administered via paper forms (53.4\%). Screening most frequently takes place at well child visits. The primary provider most commonly administers the screening, although this work is also done by nurses and medical assistants. Screening is most likely to occur during the provider encounter or when the patient is placed in a room. Social workers, followed in frequency by providers, are the most likely to follow up on positive screens. See Tables 3 through 10 for further details about clinics' screening processes.

Tables 3-10 about here 
Clinics plan to begin screening within the next three years for $23.6 \%$ of the $500 \mathrm{SDH}$ not currently being screened. Respondents said the most common barriers to screening were lack of time $(62.5 \%)$, lack of resources to address positive screens $(50.4 \%)$, inadequate training $(45.8 \%)$ and lack of provider confidence in the ability to conduct the screen (28.6\%). Only $5 \%$ of respondents said screening was not indicated in their patient population, and only $8.8 \%$ cited inadequate evidence base as a barrier to screening. See Tables 11 and 12 for further detail on plans to implement screening and barriers.

Tables 11 and 12 about here

\section{Discussion}

The AAP and APA do not only recommend screening of pediatric patients for SDH in their medical homes; they also expect medical homes to link patients to resources and coordinate care with community agencies. ${ }^{15,19,23}$ In addition to pediatric organizations, public health champions, policymakers and the business community are adding their voices to the chorus claiming that a healthier America begins with addressing the social needs of its children. ${ }^{24,25}$ Even while the evidence mounts in support of screening for social determinants of health, our study shows that implementation is lagging.

One possible explanation is that screening itself is complex. Silverstein, et al, in 2008 argued that social screening in pediatrics should comply with established screening principles including use of valid and reliable screening instruments, effective interventions for positive screens, and evidence that earlier intervention is superior to later intervention. ${ }^{18}$ Furthermore, cost, 
acceptability to patients and providers, and potential harms must be considered when implementing any screening initiative in a pediatric practice. Finally, screening should lead to improved health outcomes, not merely improved intermediate outcomes. ${ }^{26}$

A variety of tools can screen for individual SDH domains. ${ }^{15,18}$ These tools have been developed in an array of settings and have variable applicability to primary care practice. The Edinburgh Postnatal Depression Screen (EPDS) for maternal depression ${ }^{27}$ and the 2-question food insecurity screen ${ }^{28}$ are examples of validated screening instruments whose brevity and ease make them ideal for use in the outpatient setting. In contrast, the History of Victimization Form (HVF) as a screen for child maltreatment has 65 items. ${ }^{15}$ Obviously, use of a lengthy tool that screens for only a single SDH precludes routine screening for 15 domains.

In response to this inconsistency in the availability and usability of tools, health care organizations are developing comprehensive screening tools covering an array of SDH domains. $^{29}$ The Institute of Medicine has identified 11 potential SDH domains for inclusion in the electronic health record. ${ }^{29}$ Most recently, the Centers for Medicare \& Medicaid Services (CMS) has launched the Accountable Health care Communities (AHC) innovation. This 5 year \$157 million dollar initiative will test systematic screening over the five domains of housing instability, food insecurity, transportation, utility assistance and interpersonal safety utilizing the 10 item “Accountable Health Communities Screening Tool."3031

CMS chose the term health-related social needs (HRSN) rather than social determinants of health, and specifically sought to choose domains shown to affect health and not already 
systematically screened for by health care providers. ${ }^{31}$ While this project does not encompass all potential screening needs in a pediatric population, it provides an exciting, large scale opportunity to validate and implement brief, systematic, and above all usable screening tools for social needs.

Positive SDH screens call for referral and linkage to resources with follow up and care coordination. ${ }^{15,18,23}$ Medical homes might also choose alternative strategies like direct deployment of programs to at-risk populations or interventions that foster the protective potential of resilience and family support. ${ }^{18}$ Directly offering referral to services may be a beneficial intervention, since some families who screen negative still express a desire for services. ${ }^{32} \mathrm{~A}$ recent systematic review of interventions targeting SDH showed that interventions improve identification of SHD and referrals to resources. ${ }^{33}$ The actual number of patients connecting to resources is variable. Heterogeneity and variability also characterize health outcome measurements, especially in pediatric patients. ${ }^{33}$ Although evidence suggests that SDH interventions lower health care cost and utilization, this evidence comes from few studies using heterogeneous outcomes measures, with mixed results. ${ }^{33}$ If the aforementioned AHC initiative demonstrates significant cost savings, health care organizations will have increased motivation to screen. Communities participating in the AHC innovation will be placed into one of three tracks. Screening and referral will constitute the "Awareness" track. The intermediate track, "Assistance," will add service navigation, and the "Alignment" track will add structural support to ensure service provision and data analysis. ${ }^{31}$ Results of these three intervention levels will provide robust data on cost and utilization that may powerfully motivate governmental funding 
of screening. This project is designed to assess cost and health care utilization, however, the rich CMS database could also be used to examine specific health outcomes over the five year period.

Screening for SDH screening is acceptable to pediatric patients and their caregivers, and it can improve their perception of care. Families have self-reported increased satisfaction with providers, increased linkage to services, and better health care outcomes when their medical providers ask about SDH. ${ }^{21,34-36}$ On the provider side, barriers previously noted in the literature have included: lack of recognized benefit measurable outcome of screening, lack of time, lack of professional training, lack of familiarity with relevant tools, and lack of knowledge of community resources. ${ }^{1521}$ Our study indicates that providers accept the evidence and indication for screening. They hesitate primarily because of time constraints, indicating the need for streamlined screening methods, improved workflow and more use of interdisciplinary teams so that the medical provider is not working alone to address the daunting problems caused by social determinants of health. The barriers raised by lack of confidence and inadequate training can be addressed through educational activities at the resident level, and by professional outreach and continuing medical education (CME) activities for practicing pediatricians.

Little is published on the harms of SDH screening in pediatric practice. Although families accept and embrace screening, families also exhibit a spectrum of comfort and discomfort with various domains; most families, for example, believe they should not be asked about neighborhood violence by medical providers. ${ }^{21}$ Potentially, families might not present to health care settings if they are wary of being asked uncomfortable questions there. Stigma is another 
potential harm. Clinics could mitigate these harms by screening systematically rather than selectively, and by explaining the intended health benefits of the screening.

In our study, four SDH were being screened for in $60 \%$ or more of clinics. Characteristics of the most commonly screened SDH include validated, concise screening tools, longstanding Bright Futures recommendations, literature suggesting benefit, and identifiable interventions. An illustrative example is maternal depression screening. In our study, $86 \%$ of clinics reported screening for maternal depression, the highest of any domain. Maternal depression screening has been recommended as a best practice in Bright Futures since 2008. ${ }^{37}$ Additionally, the EPDS is a brief, validated screening tool for postpartum depression. ${ }^{15}$ Postpartum depression affects 10$15 \%$ of women and is amenable to treatment. ${ }^{38}$ Poor health and educational outcomes have been well documented for children of mothers with untreated depression. ${ }^{15,18}$ While mothers may only access health care for themselves at a single postpartum visit, they routinely present with their infants for multiple pediatric encounters from birth through six months, giving pediatric offices a unique opportunity to screen. Successful referral and treatment is possible in primary care settings. ${ }^{39}$ These reasons support the strong level of screening occurring in pediatric practice. Future work could bridge the remaining evidence gap, clearly linking screening for maternal depression in the pediatric setting and improved child health outcomes.

Child educational problems overlap with developmental evaluations, a traditional part of the pediatric visit that superseded the notions of systematic screening and validated tools for SDH. It logically follows that $84 \%$ of the clinics in our sample screen for education. Several validated developmental screens such as the Parents Evaluation of Developmental Status (PEDS) are 
available, as are question templates in Bright Futures specifically addressing school

performance. ${ }^{15,37}$ Education screening may have demonstrated its distinct connection to desirable outcomes: primary care interventions can increase enrollment in Headstart ${ }^{15,40}$ and enrollment in early childhood education programs improves educational and socioeconomic outcomes related to improved health outcomes and longevity. ${ }^{15,18,40-43}$ Pediatricians can facilitate referral of school aged children for the evaluation and services to which they are entitled by law, thereby potentially leading to optimal school performance. ${ }^{15}$

In addition to maternal depression and educational problems, Bright Futures has recommended inquiry on firearms in the household, household substance abuse and intimate partner violence at specified well-child visits since $2008 .{ }^{37}$ Providers are guided to ask about food insecurity if there is a growth problem. Pediatricians are to survey for signs and symptoms of child abuse, but specific questions are not included. In contrast, Bright Futures 2008 screening and counseling guidelines do not mention family financial support, the home and neighborhood environment, immigration, parental incarceration, or family stress, all domains where screening is less common.

Providers detect food insecurity, the third most commonly screened SDH, using a simple, validated two question screen ${ }^{28}$ and its use can lead to common interventions, such as referrals to WIC, SNAP and community food pantries ${ }^{15}$ It is not surprising that it is in the top tier of SDH being screened even though it did not have a universal recommendation by the AAP in 2008 . The screen was not developed in 2010. Food insecurity's relationship to a traditional biomedical 
parameter, growth, joined with a simple screen and a burgeoning body of literature on childhood obesity, make it a natural and comfortable area for pediatric inquiry.

Firearm exposure is the fourth most commonly screened domain. It lacks a validated screening tool, but questions for screening are part of Bright Futures Guidelines. ${ }^{37}$ More importantly, firearms contribute to homicide, suicide and unintentional injury, the top three causes of morbidity and mortality in the pediatric population. ${ }^{44}$ This burden disproportionately affects African American teen males. ${ }^{45}$ The possibility of mitigating preventable and disparate pediatric injury and death may motivate pediatricians to screen.

SDH being screened at an intermediate level are either traditionally recognized determinants which are difficult to address, or they are newer additions to the broadening scope of pediatric primary care supported by evidence and professional societies. For example, child maltreatment, intimate partner violence, and parental substance abuse are long recognized anticipatory guidance items, however, pediatricians may feel less comfortable inquiring about these sensitive parental behaviors. Caregivers may be reticent to report them from embarrassment or fear of legal repercussion.

Family financial support traditionally fell outside a routine pediatric encounter. ${ }^{21}$ Global acknowledgement of poverty as an upstream link to poor health, and urging from the AAP, ${ }^{23}$ have helped it to surpass child maltreatment and parental substance abuse in level of screening. Pediatricians have not traditionally screened in a systematic way for parental stress or childcare needs, however, these topics easily integrate into child-rearing discussions and are low stakes. 
Less than one third of pediatric clinics are screening for home or neighborhood environment, parental incarceration, parental health literacy or immigration status. These SDH all lie outside the traditional biomedical model, may be associated with stigma, and do not have simple or proven interventions. Of this lower tier, a validated screening tool exists only for parental health literacy. Immigration status is the least commonly screened SDH. Free text comments indicate that both providers and patients would be uncomfortable, and would fear legal repercussions if immigration status were documented.

In our study, commonly cited barriers to SDH screening are lack of time, lack of resources to address positive screens, and lack of provider education. The majority of screening is being done by the provider during the encounter, or by the nurse or MA during rooming. Even with $83 \%$ of responding clinics having a social worker present, this alone does not indicate whether the practice has the capacity to address positive screens. These findings suggest the need for innovative restructuring of medical homes. The routine use of a comprehensive screening tool administered by preclinical staff could greatly enhance workflow. Up to date local resource guides could be created and maintained by team members. Health care systems are creating innovative models using undergraduate students, pediatric residents, community partners or embedded care navigators for this laborious task. ${ }^{46}$ Increased integration of medical legal partnerships, domestic violence counselors and community health workers, currently present in less than one third of clinics, might extend the clinician's ability to connect patients to effective community resources. If the aforementioned $\mathrm{ACH}$ initiative convincingly demonstrates cost savings, it may drive funding for this additional staff if cost savings are convincingly demonstrated. 


\section{Limitations}

Our study is limited to pediatric continuity clinics in the CORNET research net and may not be representative of other types of patient centered medical homes. Even within CORNET, we were able to achieve a response rate of only $41 \%$ of CORNET member institutions and $44 \%$ of the total clinic sites participating. The layers of communication between the continuity clinic site directors and the investigators were obstacles to higher response rates and, perhaps, investigators' better understanding of how the clinics did and could operate.

Our results likely overestimate the level of screening for SDH in pediatric resident continuity clinic, since sites with an interest in screening are more likely to have responded to the survey. Also, institutional screening norms may be similar across clinic sites within the same institution, causing further overestimation of screening practices and reduction in generalizability of results.

Finally, we would postulate that resident continuity clinics who are part of the CORNET research network are more likely to be familiar with new research on SDH, and thus more likely to screen than are practices outside Academic Medical Centers.

\section{Conclusion}

Screening for Social Determinants of Health is a broad and complex topic with important implications for mitigating the effects of child poverty, adversity and toxic stress, and improving child health and adult outcomes. Despite its importance and the recommendation of the AAP 
and the APA, universal levels of screening for SDH have not yet been achieved in pediatric resident continuity clinics. Characteristics of commonly screened SDH include validated, concise screening tools, longstanding Bright Futures recommendations, literature suggesting benefit, and identifiable interventions. The major barrier to screening is lack of time. Clinicians also indicate reluctance to screen for SDH if they fear legal repercussions or embarrassment to their patients, but screening is important nonetheless, since we can only analyze and address the risk factors of which we are aware.

Opportunities for research abound in this fast-moving field. The SDH domains themselves continue to be redefined as health care organizations strive to create comprehensive screening tools. ${ }^{29}$ Further progress requires screening tools that cover multiple domains but are sensitive, valid and concise. While our understanding of the harms of adverse SDH grows, we need to continue to gather evidence showing which primary care initiatives truly improve health outcomes. Primary care initiatives to address SDH must be acceptable to provider and patient and confer greater benefit than harm.

Pediatricians in patient centered medical homes are uniquely positioned to screen and intervene on SDH, but they cannot do it alone. The CMS ACH initiative will be a unique opportunity to compile needed cost and outcome data. The products of the initiative could channel health care dollars upstream to support the formation of multidisciplinary teams, community partnerships, and policy initiatives that will work in concert with health care professionals for the good of our nation's children. 


\section{Selected References}

1. Marmot M. Closing the gap in a generation: health equity through action on the social determinants of health. The Lancet (British edition).372(9650):1661-1669.

2. Currie C ZC, Morgan A, et al. . Social Determinants of health and well-being among young people. Health Behaviour in School-aged Children (HBSC) Study. . Copenhagen, Denmark2012.

3. Egbuonu L. Child health and social status. Pediatrics (Evanston).69(5):550-557.

4. Organization WH. Closing the Gap: Policy into practice on social determinants of health (Discussion Paper). Paper presented at: World Conference on Social Determinants of Health2011; Rio de Janeiro, Brazil.

5. Jie Y. Do indoor environments influence asthma and asthma-related symptoms among adults in homes?: a review of the literature. J Formos Med Assoc.110(9):555-563.

6. Ahluwalia SK. The indoor environment and its effects on childhood asthma. Curr Opin Allergy Clin Immunol.11(2):137-143.

7. Shonkoff JP. Neuroscience, molecular biology, and the childhood roots of health disparities: building a new framework for health promotion and disease prevention. JAMA : the journal of the American Medical Association.301(21):2252-2259.

8. Shonkoff JP. The lifelong effects of early childhood adversity and toxic stress. Pediatrics (Evanston).129(1):e232-e246.

9. Felitti VJV. Relationship of childhood abuse and household dysfunction to many of the leading causes of death in adults. The Adverse Childhood Experiences (ACE) Study. American journal of preventive medicine.14(4):245-258.

10. Dong M. Insights into causal pathways for ischemic heart disease: adverse childhood experiences study. Circulation (New York, NY).110(13):1761-1766.

11. Brown DW. Adverse childhood experiences are associated with the risk of lung cancer: a prospective cohort study. BMC Public Health.10:20.

12. Nemeroff CB. Paradise Lost: The Neurobiological and Clinical Consequences of Child Abuse and Neglect. Neuron (Cambridge, Mass).89(5):892-909.

13. Semega JL, Fontenot KR, Kollar MA. Income and Poverty in the United States: 2016. Washington, DC: United States Census Bureau. 2017.

14. Federal Poverty Level (FPL). 2017; https://www.health care.gov/glossary/federal-poverty-levelFPL/. Accessed November 22, 2017, 2017.

15. Chung EK. Screening for Social Determinants of Health Among Children and Families Living in Poverty: A Guide for Clinicians. Curr Probl Pediatr Adolesc Health Care.46(5):135-153.

16. Larson K. Influence of multiple social risks on children's health. Pediatrics (Evanston).121(2):337344.

17. Garg A. Surveillance and Screening for Social Determinants of Health: The Medical Home and Beyond. JAMA pediatrics.170(3):189-190.

18. Silverstein $M$, Conroy K, Sandel M. Screening for social determinants of health in pediatric primary care. Pediatr Ann. 2008;37(11):740-746.

19. Block RW. An agenda for children for the 113th Congress: recommendations from the pediatric academic societies. Pediatrics (Evanston).131(1):109-119.

20. Poverty and Child Health in the United States. Pediatrics (Evanston).137(4).

21. Kogan MD. Routine assessment of family and community health risks: parent views and what they receive. Pediatrics (Evanston). 2004;113(6 suppl):1934-1943.

22. CORNET Continuity Research Network. https://www.academicpeds.org/research/research CORNET.cfm. Accessed November 19, 2017. 
23. Poverty and Child Health in the United States. Pediatrics (Evanston).137(4):e20160339e20160339.

24. Bixenstine P CP, and Jain SH. Fighting Chronic Disease Starts with Better Pediatric Care. Harv Bus Rev. 2017. https://hbr.org/2017/10/fighting-chronic-disease-starts-with-better-pediatric-care.

25. Heiman HJ, Artiga S. Beyond health care: the role of social determinants in promoting health and health equity. Health (N Y). 2015;20(10).

26. Harris R. Reconsidering the criteria for evaluating proposed screening programs: reflections from 4 current and former members of the U.S. Preventive services task force. Epidemiol Rev. 2011;33(1):20-35.

27. Cox JL, Holden JM, Sagovsky R. Detection of postnatal depression. Development of the 10-item Edinburgh Postnatal Depression Scale. Br J Psychiatry. 1987;150:782-786.

28. Hager ER, Quigg AM, Black MM, et al. Development and validity of a 2-item screen to identify families at risk for food insecurity. Pediatrics. 2010;126(1):e26-32.

29. LaForge K, Gold R, Cottrell E, et al. How 6 Organizations Developed Tools and Processes for Social Determinants of Health Screening in Primary Care: An Overview. J Ambul Care Manage. 2017.

30. Alley DE. Accountable Health Communities--Addressing Social Needs through Medicare and Medicaid. The New England journal of medicine. 2016;374(1):8-11.

31. Billioux A VK, Anthony S, Alley D. Standardized screening for health-related social needs in clinical settings: The accountable health communities screening tool. . 2017; https://nam.edu/wp-content/uploads/2017/05/Standardized-Screening-for-Health-RelatedSocial-Needs-in-Clinical-Settings.pdf. Accessed 11/24/2017.

32. Bottino CJ, Rhodes ET, Kreatsoulas C, Cox JE, Fleegler EW. Food Insecurity Screening in Pediatric Primary Care: Can Offering Referrals Help Identify Families in Need? Acad Pediatr. 2016.

33. Gottlieb LM. A Systematic Review of Interventions on Patients' Social and Economic Needs. American journal of preventive medicine.53(5):719-729.

34. Colvin JD, Bettenhausen JL, Anderson-Carpenter KD, Collie-Akers V, Chung PJ. Caregiver Opinion of In-Hospital Screening for Unmet Social Needs by Pediatric Residents. Acad Pediatr. 2016;16(2):161-167.

35. Colvin JD, Bettenhausen JL, Anderson-Carpenter KD, et al. Multiple Behavior Change Intervention to Improve Detection of Unmet Social Needs and Resulting Resource Referrals. Acad Pediatr. 2016;16(2):168-174.

36. Fleegler EW, Lieu TA, Wise PH, Muret-Wagstaff S. Families' health-related social problems and missed referral opportunities. Pediatrics. 2007;119(6):e1332-1341.

37. Hagan JF SJ, Duncan P, editors. 2017 Recommendations for Preventive Pediatric Health Care. Pediatrics (Evanston).139(4).

38. Liberto TL. Screening for depression and help-seeking in postpartum women during well-baby pediatric visits: an integrated review. J Pediatr Health Care.26(2):109-117.

39. Olin SC, Kerker B, Stein RE, et al. Can Postpartum Depression Be Managed in Pediatric Primary Care? Journal of women's health (2002). 2016;25(4):381-390.

40. Silverstein M, Mack C, Reavis N, Koepsell TD, Gross GS, Grossman DC. Effect of a clinic-based referral system to head start: a randomized controlled trial. JAMA. 2004;292(8):968-971.

41. Anderson LM, Shinn C, Fullilove MT, et al. The effectiveness of early childhood development programs. A systematic review. Am J Prev Med. 2003;24(3 Suppl):32-46.

42. Schweinhart L. Significant Benefits: The High/Scope Perry Preschool Study through Age 27. Monographs of the High/Scope Educational Research Foundation, No. Ten. ERIC; 1993. 
43. Love JM, Kisker EE, Ross CM, et al. Making a Difference in the Lives of Infants and Toddlers and Their Families: The Impacts of Early Head Start. Volumes I-III: Final Technical Report [and] Appendixes [and] Local Contributions to Understanding the Programs and Their Impacts. 2002.

44. Heron MP. Deaths: leading causes for 2012. 2015.

45. Fowler KA, Dahlberg LL, Haileyesus T, Gutierrez C, Bacon S. Childhood Firearm Injuries in the United States. Pediatrics. 2017;140(1).

46. Fazalullasha F, Taras J, Morinis J, et al. From office tools to community supports: The need for infrastructure to address the social determinants of health in paediatric practice. Paediatr Child Health. 2014;19(4):195-199. 
Figure 1

Clinics Screening for Each SDH

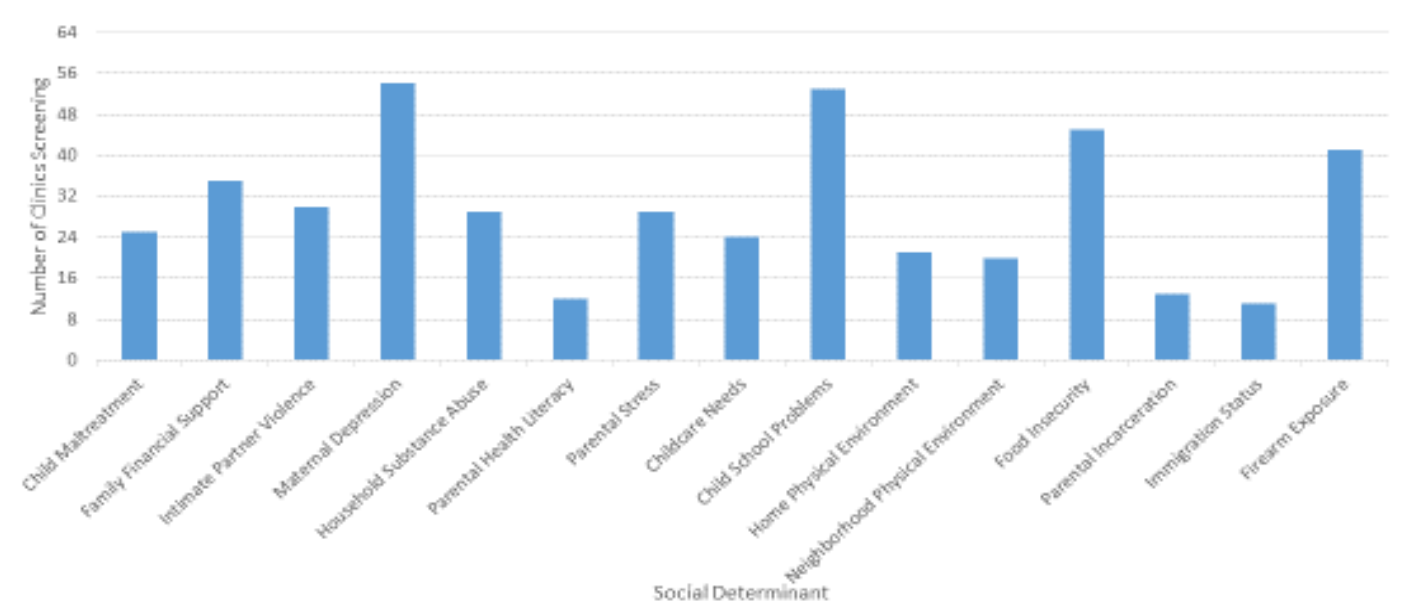


Table 1. Demographics of Participating Clinics

\begin{tabular}{|c|c|}
\hline Clinic Characteristic & Mean (range) \\
\hline Number of Residents & $73 \quad(12-190)$ \\
\hline$\%$ Residents Entering Primary Care & $41 \quad(0-100)$ \\
\hline Available Clinic Sites & $(1-39)$ \\
\hline Annual Patients Served & $9477 \quad(1,000-39,439)$ \\
\hline Annual Encounters & $18727 \quad(2,700-80,000)$ \\
\hline$\%$ Medicaid & $(0-98)$ \\
\hline$\%$ Hispanic & $28 \quad(0-80)$ \\
\hline$\%$ Black & $(2-93)$ \\
\hline \%White & $(1-91)$ \\
\hline Region of country located: & Percent/Number $(n=64)$ \\
\hline Northeast & $23.4 \%(15)$ \\
\hline Midwest & $17.2 \%(11)$ \\
\hline South & $43.8 \%(28)$ \\
\hline West & $15.6 \%(10)$ \\
\hline Type & Percent/Number $(n=64)$ \\
\hline Social Worker & $83 \%(53)$ \\
\hline Social Work Surrogate & $61 \%(39)$ \\
\hline Domestic Violence Counselor & $20 \%(13)$ \\
\hline Community Health Worker & $34 \%(22)$ \\
\hline Medical Legal Partnership & $33 \%(21)$ \\
\hline
\end{tabular}




\section{Table 2}

Summary of Social Determinant Screening

\begin{tabular}{|l|l|l|l|l|}
\hline Determinant & Total N & $\begin{array}{l}\text { Screening } \\
(\# / \%)\end{array}$ & $\begin{array}{l}\text { Not Screening } \\
(\# / \%)\end{array}$ & $\begin{array}{l}\text { Missing } \\
(\# / \%)\end{array}$ \\
\hline Child Maltreatment & 64 & $25 / 40$ & $37 / 60$ & 2 \\
\hline Family Financial Support & 64 & $35 / 56$ & $28 / 44$ & 1 \\
\hline Intimate Partner Violence & 64 & $30 / 48$ & $33 / 52$ & 1 \\
\hline $\begin{array}{l}\text { Maternal Depression/Family Mental } \\
\text { Illness }\end{array}$ & 64 & $54 / 86$ & $9 / 14$ & 1 \\
\hline Household Substance Abuse & 64 & $29 / 46$ & $34 / 54$ & 1 \\
\hline Parental Health Literacy & 64 & $12 / 19$ & $51 / 81$ & 1 \\
\hline Parental Stress & 64 & $29 / 47$ & $33 / 53$ & 2 \\
\hline Childcare Needs & 64 & $24 / 39$ & $38 / 61$ & 2 \\
\hline Child Education/ School Problems & 64 & $53 / 84$ & $10 / 16$ & 1 \\
\hline Home Physical Environment & 64 & $21 / 33$ & $42 / 67$ & 1 \\
\hline Neighborhood Physical Environment & 64 & $20 / 32$ & $43 / 68$ & 1 \\
\hline Food Insecurity & 64 & $45 / 71$ & $18 / 29$ & 1 \\
\hline Parental Incarceration & 64 & $13 / 21$ & $50 / 79$ & 1 \\
\hline Immigration Status & 64 & $11 / 17$ & $52 / 83$ & 1 \\
\hline Firearm Exposure & 64 & $41 / 65$ & $22 / 35$ & 1 \\
\hline TOTALS & 960 & $442 / 46$ & $500 / 52$ & $18 / 2$ \\
\hline
\end{tabular}


Table 3 Source of Screening Tool for each SDH

\begin{tabular}{|c|c|c|c|c|c|c|}
\hline Determinant & $\begin{array}{l}\text { Screening } \\
(\# / \%)\end{array}$ & $\begin{array}{l}\text { Validated } \\
\text { tool (\#) }\end{array}$ & $\begin{array}{l}\text { Adapted } \\
\text { Tool (\#) }\end{array}$ & $\begin{array}{l}\text { Original } \\
\text { Tool (\#) }\end{array}$ & $\begin{array}{l}\text { Unsure o } \\
(\#)\end{array}$ & $\begin{array}{l}\text { Missing } \\
(\#)\end{array}$ \\
\hline $\begin{array}{l}\text { Child } \\
\text { Maltreatment }\end{array}$ & $25 / 40$ & 7 & 3 & 6 & 9 & 0 \\
\hline $\begin{array}{l}\text { Family } \\
\text { Financial } \\
\text { Support }\end{array}$ & $35 / 56$ & 10 & 6 & 13 & 5 & 1 \\
\hline $\begin{array}{l}\text { Intimate } \\
\text { Partner } \\
\text { Violence }\end{array}$ & $30 / 47$ & 10 & 5 & 9 & 6 & 0 \\
\hline $\begin{array}{l}\text { Maternal } \\
\text { Depression }\end{array}$ & $54 / 85$ & 44 & 5 & 4 & 1 & 0 \\
\hline $\begin{array}{l}\text { Household } \\
\text { Substance } \\
\text { Abuse }\end{array}$ & $29 / 46$ & 13 & 4 & 5 & 7 & 0 \\
\hline $\begin{array}{l}\text { Parental } \\
\text { Health } \\
\text { Literacy }\end{array}$ & $12 / 19$ & 1 & 2 & 3 & 6 & 0 \\
\hline $\begin{array}{l}\text { Parental } \\
\text { Stress }\end{array}$ & $29 / 47$ & 8 & 4 & 8 & 9 & 0 \\
\hline $\begin{array}{l}\text { Childcare } \\
\text { Needs }\end{array}$ & $24 / 39$ & 2 & 4 & 8 & 9 & 1 \\
\hline $\begin{array}{l}\text { Child School } \\
\text { Problems }\end{array}$ & $53 / 84$ & 12 & 4 & 17 & 20 & 0 \\
\hline $\begin{array}{l}\text { Home } \\
\text { Physical } \\
\text { Environment }\end{array}$ & $21 / 33$ & 4 & 4 & 9 & 4 & 0 \\
\hline $\begin{array}{l}\text { Neighborhood } \\
\text { Physical } \\
\text { Environment }\end{array}$ & $20 / 32$ & 1 & 3 & 12 & 4 & 0 \\
\hline $\begin{array}{l}\text { Food } \\
\text { Insecurity }\end{array}$ & $45 / 71$ & 25 & 9 & 7 & 4 & 0 \\
\hline $\begin{array}{l}\text { Parental } \\
\text { Incarceration }\end{array}$ & $13 / 21$ & 0 & 2 & 7 & 4 & 0 \\
\hline $\begin{array}{l}\text { Immigration } \\
\text { Status }\end{array}$ & $11 / 17$ & 0 & 1 & 7 & 3 & 0 \\
\hline $\begin{array}{l}\text { Firearm } \\
\text { Exposure }\end{array}$ & $41 / 65$ & 4 & 4 & 14 & 19 & 0 \\
\hline TOTAL & 442 & $141(31.9 \%)$ & $61(13.8 \%)$ & $202(45.7 \%)$ & $111(25.1 \%)$ & $2(0.45 \%)$ \\
\hline
\end{tabular}


Table 4 Visit Type When SDH Screening Occurs

\begin{tabular}{|l|l|l|l|l|l|l|l|}
\hline Determinant & $\begin{array}{l}\text { Screeni } \\
\text { ng } \\
\text { (\#/\%) }\end{array}$ & $\begin{array}{l}\text { All } \\
\text { visits }\end{array}$ & $\begin{array}{l}\text { All well } \\
\text { visits }\end{array}$ & $\begin{array}{l}\text { New } \\
\text { visits }\end{array}$ & $\begin{array}{l}\text { Provider } \\
\text { led }\end{array}$ & $\begin{array}{l}\text { Specific } \\
\text { Well Visits }\end{array}$ & Other \\
\hline $\begin{array}{l}\text { Child } \\
\text { Maltreatment }\end{array}$ & $25 / 40$ & 3 & 11 & 0 & 8 & 7 & 1 \\
\hline $\begin{array}{l}\text { Family } \\
\text { Financial } \\
\text { Support }\end{array}$ & $35 / 56$ & 6 & 17 & 1 & 7 & 9 & 2 \\
\hline $\begin{array}{l}\text { Intimate } \\
\text { Partner } \\
\text { Violence }\end{array}$ & $30 / 47$ & 5 & 13 & 0 & 6 & 8 & 3 \\
\hline $\begin{array}{l}\text { Maternal } \\
\text { Depression }\end{array}$ & $54 / 85$ & 1 & 9 & 1 & 5 & 36 & 8 \\
\hline $\begin{array}{l}\text { Household } \\
\text { Substance } \\
\text { Abuse }\end{array}$ & $29 / 46$ & 3 & 11 & 0 & 10 & 9 & 4 \\
\hline $\begin{array}{l}\text { Parental } \\
\text { Health } \\
\text { Literacy }\end{array}$ & $12 / 19$ & 2 & 1 & 2 & 1 & 6 & 0 \\
\hline $\begin{array}{l}\text { Parental } \\
\text { Stress }\end{array}$ & $29 / 47$ & 1 & 14 & 0 & 9 & 10 & 3 \\
\hline $\begin{array}{l}\text { Childcare } \\
\text { Needs }\end{array}$ & $24 / 39$ & 3 & 11 & 0 & 7 & 8 & 1 \\
\hline $\begin{array}{l}\text { Child School } \\
\text { Problems }\end{array}$ & $53 / 84$ & 2 & 39 & 1 & 13 & 10 & 1 \\
\hline $\begin{array}{l}\text { Home } \\
\text { Physical } \\
\text { Environment }\end{array}$ & $21 / 33$ & 4 & 9 & 0 & 3 & 7 & 1 \\
\hline $\begin{array}{l}\text { Neighborhoo } \\
\text { d Physical } \\
\text { Environment }\end{array}$ & $20 / 32$ & 3 & 9 & 0 & 4 & 5 & 1 \\
\hline $\begin{array}{l}\text { Food } \\
\text { Insecurity }\end{array}$ & $45 / 71$ & 6 & 22 & 0 & 8 & 15 & 3 \\
\hline $\begin{array}{l}\text { Parental } \\
\text { Incarceration }\end{array}$ & $13 / 21$ & 3 & 2 & 0 & 3 & 6 & 3 \\
\hline $\begin{array}{l}\text { Immigration } \\
\text { Status }\end{array}$ & $11 / 17$ & 2 & 4 & 0 & 3 & 2 & $33(7 \%)$ \\
\hline $\begin{array}{l}\text { Firearm } \\
\text { Exposure }\end{array}$ & $41 / 65$ & 1 & 27 & 4 & 7 & 8 & \\
\hline Totals & 442 & $45(10 \%)$ & $199(45 \%)$ & $9(2 \%)$ & $94(21 \%)$ & $146(33 \%)$ & \\
\hline
\end{tabular}


Table 5 Method of Screening Administration

\begin{tabular}{|c|c|c|c|c|c|c|}
\hline Determinant & $\begin{array}{l}\text { Screeni } \\
\text { ng } \\
(\# / \%)\end{array}$ & $\begin{array}{l}\text { Face to } \\
\text { face }\end{array}$ & paper & electronic & other & Missing \\
\hline Child Maltreatment & $25 / 40$ & 9 & 14 & 1 & 1 & 0 \\
\hline $\begin{array}{l}\text { Family Financial } \\
\text { Support }\end{array}$ & $35 / 56$ & 8 & 24 & 2 & 1 & 0 \\
\hline $\begin{array}{l}\text { Intimate Partner } \\
\text { Violence }\end{array}$ & $30 / 47$ & 8 & 20 & 1 & 1 & 0 \\
\hline Maternal Depression & $54 / 85$ & 5 & 42 & 4 & 2 & 1 \\
\hline $\begin{array}{l}\text { Household } \\
\text { Substance Abuse }\end{array}$ & $29 / 46$ & 10 & 17 & 1 & 1 & 0 \\
\hline $\begin{array}{l}\text { Parental Health } \\
\text { Literacy }\end{array}$ & $12 / 19$ & 6 & 5 & 0 & 1 & 0 \\
\hline Parental Stress & $29 / 47$ & 13 & 14 & 1 & 1 & 0 \\
\hline Childcare Needs & $24 / 39$ & 9 & 13 & 1 & 1 & 0 \\
\hline $\begin{array}{l}\text { Child School } \\
\text { Problems }\end{array}$ & $53 / 84$ & 35 & 15 & 2 & 1 & 0 \\
\hline $\begin{array}{l}\text { Home Physical } \\
\text { Environment }\end{array}$ & $21 / 33$ & 4 & 14 & 2 & 1 & 0 \\
\hline $\begin{array}{l}\text { Neighborhood } \\
\text { Physical } \\
\text { Environment }\end{array}$ & $20 / 32$ & 7 & 9 & 1 & 1 & 2 \\
\hline Food Insecurity & $45 / 71$ & 9 & 31 & 3 & 1 & 1 \\
\hline $\begin{array}{l}\text { Parental } \\
\text { Incarceration }\end{array}$ & $13 / 21$ & 8 & 4 & 0 & 1 & 0 \\
\hline Immigration Status & $11 / 17$ & 4 & 6 & 0 & 1 & 0 \\
\hline Firearm Exposure & $41 / 65$ & 27 & 8 & 2 & 2 & 2 \\
\hline Totals & 442 & $162(37 \%)$ & $236(53 \%)$ & $21(5 \%)$ & $17(4 \%)$ & $6(1 \%)$ \\
\hline
\end{tabular}


Table 6 Staff Member Administering Screen

\begin{tabular}{|l|l|l|l|l|l|l|}
\hline Determinant & $\begin{array}{l}\text { Screening } \\
(\# / \%)\end{array}$ & $\begin{array}{l}\text { Preclinical } \\
\text { staff }\end{array}$ & Nurse/MA & $\begin{array}{l}\text { Primary } \\
\text { Provider }\end{array}$ & $\begin{array}{l}\text { Social } \\
\text { Worker }\end{array}$ & Other \\
\hline $\begin{array}{l}\text { Child } \\
\text { Maltreatment }\end{array}$ & $25 / 40$ & 8 & 12 & 10 & 2 & 0 \\
\hline $\begin{array}{l}\text { Family } \\
\text { Financial } \\
\text { Support }\end{array}$ & $35 / 56$ & 12 & 16 & 15 & 1 & 0 \\
\hline $\begin{array}{l}\text { Intimate Partner } \\
\text { Violence }\end{array}$ & $30 / 47$ & 7 & 16 & 13 & 4 & 0 \\
\hline $\begin{array}{l}\text { Maternal } \\
\text { Depression }\end{array}$ & $54 / 85$ & 20 & 29 & 14 & 4 & 0 \\
\hline $\begin{array}{l}\text { Household } \\
\text { Substance } \\
\text { Abuse }\end{array}$ & $29 / 46$ & 7 & 10 & 17 & 2 & 0 \\
\hline $\begin{array}{l}\text { Parental Health } \\
\text { Literacy }\end{array}$ & $12 / 19$ & 5 & 6 & 4 & 0 & 0 \\
\hline Parental Stress & $29 / 47$ & 7 & 12 & 15 & 3 & 0 \\
\hline $\begin{array}{l}\text { Childcare } \\
\text { Needs }\end{array}$ & $24 / 39$ & 7 & 7 & 11 & 1 & 0 \\
\hline $\begin{array}{l}\text { Child School } \\
\text { Problems }\end{array}$ & $53 / 84$ & 7 & 11 & 38 & 2 & 0 \\
\hline $\begin{array}{l}\text { Home Physical } \\
\text { Environment }\end{array}$ & $21 / 33$ & 6 & 10 & 8 & 1 & 0 \\
\hline $\begin{array}{l}\text { Neighborhood } \\
\text { Physical } \\
\text { Environment }\end{array}$ & $20 / 32$ & 5 & 5 & 12 & 2 & 0 \\
\hline \begin{tabular}{l} 
Food Insecurity \\
\hline Parental \\
Incarceration
\end{tabular} & $13 / 71$ & 14 & 20 & 20 & 1 & 0 \\
\hline $\begin{array}{l}\text { Immigration } \\
\text { Status }\end{array}$ & $11 / 17$ & 3 & 5 & 9 & 1 & 0 \\
\hline $\begin{array}{l}\text { Firearm } \\
\text { Exposure }\end{array}$ & $41 / 65$ & 1 & 54 & 1 & 0 \\
\hline
\end{tabular}


Table 7 Timing of SDH Screening

\begin{tabular}{|c|c|c|c|c|c|c|}
\hline Determinant & $\begin{array}{l}\text { Screening } \\
(\# / \%)\end{array}$ & $\begin{array}{l}\text { Prior to } \\
\text { Encounter } \\
\text { Date }\end{array}$ & Reg/WR & Rooming & Encounter & Missing \\
\hline $\begin{array}{l}\text { Child } \\
\text { Maltreatment }\end{array}$ & $25 / 40$ & 0 & 6 & 11 & 8 & 0 \\
\hline $\begin{array}{l}\text { Family } \\
\text { Financial } \\
\text { Support }\end{array}$ & $35 / 56$ & 0 & 9 & 14 & 11 & 1 \\
\hline $\begin{array}{l}\text { Intimate Partner } \\
\text { Violence }\end{array}$ & $30 / 47$ & 0 & 7 & 14 & 9 & 00 \\
\hline $\begin{array}{l}\text { Maternal } \\
\text { Depression }\end{array}$ & $54 / 85$ & 0 & 16 & 24 & 14 & 0 \\
\hline $\begin{array}{l}\text { Household } \\
\text { Substance } \\
\text { Abuse }\end{array}$ & $29 / 46$ & 0 & 6 & 10 & 3 & 0 \\
\hline $\begin{array}{l}\text { Parental Health } \\
\text { Literacy }\end{array}$ & $12 / 19$ & 0 & 5 & 4 & 3 & 0 \\
\hline Parental Stress & $29 / 47$ & 0 & 6 & 9 & 14 & 0 \\
\hline Childcare Needs & $24 / 39$ & 0 & 6 & 8 & 10 & 0 \\
\hline $\begin{array}{l}\text { Child School } \\
\text { Problems }\end{array}$ & $53 / 84$ & 0 & 6 & 8 & 39 & 0 \\
\hline $\begin{array}{l}\text { Home Physical } \\
\text { Environment }\end{array}$ & $21 / 33$ & 0 & 5 & 10 & 6 & 0 \\
\hline $\begin{array}{l}\text { Neighborhood } \\
\text { Physical } \\
\text { Environment }\end{array}$ & $20 / 32$ & 0 & 5 & 6 & 9 & 0 \\
\hline Food Insecurity & $45 / 71$ & 0 & 11 & 19 & 15 & 0 \\
\hline $\begin{array}{l}\text { Parental } \\
\text { Incarceration }\end{array}$ & $13 / 21$ & 0 & 3 & 1 & 9 & 0 \\
\hline $\begin{array}{l}\text { Immigration } \\
\text { Status }\end{array}$ & $11 / 17$ & 0 & 3 & 3 & 5 & 0 \\
\hline $\begin{array}{l}\text { Firearm } \\
\text { Exposure }\end{array}$ & $41 / 65$ & 0 & 4 & 7 & 30 & 0 \\
\hline
\end{tabular}


Table 8 Staff Member Following Up Screening

\begin{tabular}{|c|c|c|c|c|c|c|}
\hline Determinant & $\begin{array}{l}\text { Screening } \\
(\# / \%)\end{array}$ & Nurse & $\begin{array}{l}\text { Physician } \\
\text { or APP }\end{array}$ & $\begin{array}{l}\text { Social } \\
\text { Worker }\end{array}$ & Psychologist & Other \\
\hline $\begin{array}{l}\text { Child } \\
\text { Maltreatment }\end{array}$ & $25 / 40$ & 8 & 12 & 10 & 2 & 0 \\
\hline $\begin{array}{l}\text { Family } \\
\text { Financial } \\
\text { Support }\end{array}$ & $35 / 56$ & 12 & 16 & 15 & 1 & 0 \\
\hline $\begin{array}{l}\text { Intimate Partner } \\
\text { Violence }\end{array}$ & $30 / 47$ & 7 & 16 & 13 & 4 & 0 \\
\hline $\begin{array}{l}\text { Maternal } \\
\text { Depression }\end{array}$ & $54 / 85$ & 20 & 29 & 14 & 4 & 0 \\
\hline $\begin{array}{l}\text { Household } \\
\text { Substance } \\
\text { Abuse }\end{array}$ & $29 / 46$ & 7 & 10 & 17 & 2 & 0 \\
\hline $\begin{array}{l}\text { Parental Health } \\
\text { Literacy }\end{array}$ & $12 / 19$ & 5 & 6 & 4 & 0 & 0 \\
\hline Parental Stress & $29 / 47$ & 7 & 12 & 15 & 3 & 0 \\
\hline $\begin{array}{l}\text { Childcare } \\
\text { Needs }\end{array}$ & $24 / 39$ & 7 & 7 & 11 & 1 & 0 \\
\hline $\begin{array}{l}\text { Child School } \\
\text { Problems }\end{array}$ & $53 / 84$ & 7 & 11 & 38 & 2 & 0 \\
\hline $\begin{array}{l}\text { Home Physical } \\
\text { Environment }\end{array}$ & $21 / 33$ & 6 & 10 & 8 & 1 & 0 \\
\hline $\begin{array}{l}\text { Neighborhood } \\
\text { Physical } \\
\text { Environment }\end{array}$ & $20 / 32$ & 5 & 5 & 12 & 2 & 0 \\
\hline Food Insecurity & $45 / 71$ & 14 & 20 & 20 & 1 & 0 \\
\hline $\begin{array}{l}\text { Parental } \\
\text { Incarceration }\end{array}$ & $13 / 21$ & 3 & 5 & 9 & 1 & 0 \\
\hline $\begin{array}{l}\text { Immigration } \\
\text { Status }\end{array}$ & $11 / 17$ & 3 & 5 & 6 & 1 & 0 \\
\hline $\begin{array}{l}\text { Firearm } \\
\text { Exposure }\end{array}$ & $41 / 65$ & 1 & 7 & 34 & 1 & 0 \\
\hline
\end{tabular}


Table 9 Plan to Screen in next 3 years

\begin{tabular}{|c|c|c|c|}
\hline Determinant & $\begin{array}{l}\text { Not } \\
\text { Screening } \\
\text { (\#) }\end{array}$ & $\begin{array}{l}\text { Plan to } \\
\text { Screen } \\
(\# / \%)\end{array}$ & Missing \\
\hline $\begin{array}{l}\text { Child } \\
\text { Maltreatment }\end{array}$ & 37 & $14 / 38$ & 0 \\
\hline $\begin{array}{l}\text { Family Financial } \\
\text { Support }\end{array}$ & 28 & $10 / 36$ & 0 \\
\hline $\begin{array}{l}\text { Intimate Partner } \\
\text { Violence }\end{array}$ & 33 & $13 / 39$ & 0 \\
\hline $\begin{array}{l}\text { Maternal } \\
\text { Depression }\end{array}$ & 9 & $6 / 67$ & 0 \\
\hline $\begin{array}{l}\text { Household } \\
\text { Substance Abuse }\end{array}$ & 34 & $9 / 26$ & 0 \\
\hline $\begin{array}{l}\text { Parental Health } \\
\text { Literacy }\end{array}$ & 51 & $8 / 16$ & 0 \\
\hline Parental Stress & 33 & $10 / 30$ & 0 \\
\hline Childcare Needs & 38 & $6 / 16$ & 0 \\
\hline $\begin{array}{l}\text { Child School } \\
\text { Problems }\end{array}$ & 10 & $1 / 11$ & 1 \\
\hline $\begin{array}{l}\text { Home Physical } \\
\text { Environment }\end{array}$ & 42 & $9 / 22$ & 1 \\
\hline $\begin{array}{l}\text { Neighborhood } \\
\text { Physical } \\
\text { Environment }\end{array}$ & 43 & $6 / 14$ & 1 \\
\hline Food Insecurity & 18 & $8 / 44$ & 0 \\
\hline $\begin{array}{l}\text { Parental } \\
\text { Incarceration }\end{array}$ & 50 & $7 / 14$ & 1 \\
\hline $\begin{array}{l}\text { Immigration } \\
\text { Status }\end{array}$ & 52 & $6 / 12$ & 1 \\
\hline $\begin{array}{l}\text { Firearm } \\
\text { Exposure }\end{array}$ & 22 & $5 / 23$ & 0 \\
\hline TOTALS & 500 & 118 & 5 \\
\hline
\end{tabular}


Table 10 Barriers to SDH Screening

\begin{tabular}{|l|l|l|l|l|l|l|l|l|}
\hline Determinant & $\begin{array}{l}\text { Not } \\
\text { Screening } \\
(\# / \%)\end{array}$ & $\begin{array}{l}\text { Lack } \\
\text { of } \\
\text { time }\end{array}$ & $\begin{array}{l}\text { Inadequate } \\
\text { training }\end{array}$ & $\begin{array}{l}\text { Lack of } \\
\text { provider } \\
\text { confidence }\end{array}$ & $\begin{array}{l}\text { Lack of } \\
\text { resources } \\
\text { to } \\
\text { address } \\
\text { positive } \\
\text { screen }\end{array}$ & $\begin{array}{l}\text { NI in } \\
\text { Patient } \\
\text { Population }\end{array}$ & $\begin{array}{l}\text { Lack of } \\
\text { Evidence }\end{array}$ & other \\
\hline $\begin{array}{l}\text { Child } \\
\text { Maltreatment }\end{array}$ & $37 / 60$ & 24 & 18 & 10 & 12 & 1 & 7 & 5 \\
\hline $\begin{array}{l}\text { Family } \\
\text { Financial } \\
\text { Support }\end{array}$ & $28 / 44$ & 18 & 18 & 9 & 17 & 0 & 2 & 5 \\
\hline $\begin{array}{l}\text { Intimate } \\
\text { Partner } \\
\text { Violence }\end{array}$ & $33 / 52$ & 20 & 18 & 15 & 13 & 2 & 3 & 5 \\
\hline $\begin{array}{l}\text { Maternal } \\
\text { Depression }\end{array}$ & $9 / 14$ & 4 & 4 & 1 & 3 & 01 & 1 & 5 \\
\hline $\begin{array}{l}\text { Household } \\
\text { Substance } \\
\text { Abuse }\end{array}$ & $34 / 54$ & 24 & 16 & 14 & 19 & 1 & 3 & 3 \\
\hline $\begin{array}{l}\text { Parental } \\
\text { Health } \\
\text { Literacy }\end{array}$ & $51 / 81$ & 35 & 24 & 14 & 25 & 1 & 3 & 7 \\
\hline $\begin{array}{l}\text { Parental } \\
\text { Stress }\end{array}$ & $33 / 53$ & 25 & 18 & 13 & 17 & 0 & 2 & 1 \\
\hline $\begin{array}{l}\text { Childcare } \\
\text { Needs }\end{array}$ & $38 / 61$ & 24 & 14 & 11 & 24 & 13 & 3 & 4 \\
\hline $\begin{array}{l}\text { Child School } \\
\text { Problems }\end{array}$ & $10 / 16$ & 8 & 4 & 3 & 3 & 0 & 1 & 1 \\
\hline $\begin{array}{l}\text { Home } \\
\text { Physical } \\
\text { Environment }\end{array}$ & $42 / 67$ & 32 & 17 & 11 & 27 & 0 & 2 & 1 \\
\hline $\begin{array}{l}\text { Neighborhood } \\
\text { Physical } \\
\text { Environment }\end{array}$ & $43 / 68$ & 28 & 19 & 12 & 28 & 0 & 6 & 1 \\
\hline $\begin{array}{l}\text { Food } \\
\text { Insecurity }\end{array}$ & $18 / 29$ & 14 & 10 & 6 & 10 & 0 & 0 & 2 \\
\hline $\begin{array}{l}\text { Parental } \\
\text { Incarceration }\end{array}$ & $50 / 79$ & 34 & 18 & 3 & 25 & 2 & 7 & 4 \\
\hline $\begin{array}{l}\text { Immigration } \\
\text { Status }\end{array}$ & $52 / 83$ & 24 & 23 & 13 & 22 & 4 & 4 & 19 \\
\hline $\begin{array}{l}\text { Firearm } \\
\text { Exposure }\end{array}$ & $22 / 35$ & 12 & 8 & 8 & 7 & 0 & 0 & \\
\hline
\end{tabular}




\section{APPENDIX A: Survey Instrument}

\section{Cornet SDH Survey}

Please complete the survey below.

Thank you!

We are studying current practices for identifying and addressing social determinants of health in pediatric resident continuity clinics enrolled in the CORNET network. We recognize that each clinic has unique patient populations, provider teams, and resource availability that influence current screening practices. We do not expect that all clinics are screening for all or most social determinants. Please provide the most accurate information available.

This study was reviewed by the University of North Carolina IRB and was determined to be IRB exempt. Data will not be reported for specific continuity clinics, residency programs, or individuals.

The initial questions request general information about the pediatric residency program at your institution.

What is the name of your residency program?

What is the total number of current residents in your program? Please include residents in all three years of pediatric training, med-peds residents, and residents in other pediatric tracks.

Does your residency have a distinct curricular track that is separate from the categorical program designed for residents planning to enter primary care?

What percentage of the residents who graduated from your program in June 2016 entered primary care?

How many continuity clinic sites are available to the residents in your residency program?

The remaining questions in this survey pertain only to residents, providers, patients, and procedures at YOUR SPECIFIC CONTINUITY CLINIC SITE.

What is the name of your continuity clinic site?

What is the zip code of your continuity clinic site?

How many of the current residents in your program have their continuity clinic at this site?

Do you have primary care track residents at this site? 
Please answer the following questions using data from academic year JULY 1, 2015 - JUNE 30, 2016. Please enter integers only. If exact numbers are not available, estimates are sufficient. If you require additional time for data acquisition, you may save and return to the survey.

What was the total number of patients served in the last year by this continuity clinic site?

What was the total number of physician/nurse practitioner encounters in the last year at this continuity clinic site? Please exclude nurse visits/immunization only visits. If specialists are embedded in your practice, please exclude those visits. Please include only primary care visits.

What percentage of annual patient visits were reimbursed by Medicaid/CHIP?

What percentage of annual patient visits were reimbursed by private insurance?

What percentage of annual patient visits were self-paid/uninsured?

Race/Ethnicity: What percentage of your patient population from July 1, 2015 to June 30, 2016 was: (Please enter integers only. No \% sign).

Hispanic or Latino?

American Indian or Alaska Native?

Asian?

Black or African American?

Native Hawaiian or other Pacific Islander?

White or Caucasian?

The next section addresses support services CURRENTLY available to your clinic site.

$\begin{array}{ll}\begin{array}{l}\text { Does this continuity clinic site currently have an } \\ \text { on-site social worker? }\end{array} & \text { Yes } \\ \begin{array}{l}\text { Does this continuity clinic site currently have an } \\ \text { on-site staff person similar to a social worker such } \\ \text { as a care manager or outreach specialist? }\end{array} & \text { Yes } \\ \begin{array}{l}\text { Does this continuity clinic site currently have a } \\ \text { domestic violence counselor available to the }\end{array} & \bigcirc \text { Yes } \\ \text { patients/families either by phone or on-site? } & \bigcirc \text { No }\end{array}$


A community health worker is a front-line public health worker who is a trusted member of and/or has

an unusually close understanding of the community

No served. Does this continuity clinic site currently collaborate with one or more community health workers?

A medical legal partnership formally embeds legal services into care to improve patient services and outcomes. An attorney may be available to the clinic population on-site or remotely. Does this continuity clinic site currently have a medical legal

partnership?

For each listed social determinant of health: (1) Indicate whether the residents at your clinic site receive specific didactic education (such as mandatory lectures, online modules, or in-services) on its effect on child development and lifelong health. (2) Indicate whether the residents receive specific skills training (such as shadowing social workers during screening, role playing, or observed encounters with feedback) on how to identify each listed social determinant of health.

Child maltreatment

Poverty/family financial support

Intimate partner violence

Maternal depression/family mental illness

Household substance abuse

Parental health literacy

Parental stress

Childcare needs

Child learning

problems/academic achievement

Physical environment -home (i.e. heat, water, rodents, mold, lead paint)

Physical environment

-neighborhood (i.e. green

spaces, grocery stores, safety,

transportation)

Food insecurity

Parental incarceration

Immigration

Firearm exposure
Didactic

Skills Training

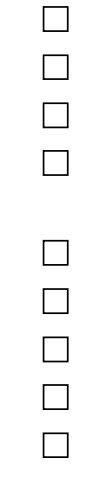

Yes

No 
Resilience (i.e. social support, parental confidence, shared rituals)

Please provide any additional comments on resident education and training regarding the impact of social determinants on patient health:

Screening is a strategy used to identify the presence of an otherwise unknown problem in a patient to reduce adverse outcomes associated with that problem. For the purpose of our survey, social determinant screening refers to asking prescribed questions in a systematic way such as through a screening tool or questions embedded in the medical record.

For each social determinant, we included a sample question for illustrative purposes. You may be screening for this category with a very different question; if so, please answer "yes" to indicate that you are screening for that topic.

We are also interested in the source of your screening questions. If you do not know, that is ok. We recognize you may ask questions covering multiple topics from several sources on one questionnaire. If possible please email a copy of the screening questionnaire(s) you are using to: Marcia.Morgenlander@unchealth.unc.edu.

Are patients/families at this continuity clinic site systematically screened for child maltreatment?

(Example screening question: Do you worry that your child may have been physically abused?)

What is the source of the screening questions on child maltreatment?

Please name validated tool.

At what types of visits is screening for child maltreatment conducted? (choose all that apply)

Please describe other visit type.

What method is used to screen for child maltreatment?

Please describe other method. $\bigcirc$ Yes

$\bigcirc$ No

a validated screening tool

an adaptation of a validated screening tool

an internally developed tool

unsure of original source

all visits

$\square$ all well child visits

$\square$ new patient visits only

$\square$ when provider feels screening is indicated

$\square$ at specifically scheduled well child visits

other

face-to-face interview

paper form

electronic questionnaire

other 
Who introduces the screening process for child maltreatment? (choose all that apply)

Please note title of other person responsible for introducing the screening process.

When does the screening for child maltreatment occur?

Who is responsible for followup of screening for child maltreatment? (choose all that apply)

Please note title of other person responsible for followup.

Do you plan to begin screening for child maltreatment within the next three years?

Please describe the current plan for screening.

What current barriers prevent screening for child maltreatment? (choose all that apply)

Please describe other barriers to screening for child maltreatment.

Are patients/families at this continuity clinic site systematically screened for financial problems/poverty?

(Example screening question: Does you family have enough money at the end of the month?)

What is the source of the screening questions on family financial support?

Please name validated tool.

At what types of visits is screening for financial support conducted? (choose all that apply)

Please describe other visit type. preclinical staff

nurse/medical assistant

$\square$ physician/ primary provider

social worker

$\square$ other

prior to the encounter date

on day of encounter at registration or in waiting room

on day of encounter during triage or rooming

during the encounter

nurse

physician/primary provider

social worker

psychologist

other

Yes

No

$\square$ lack of time

inadequate training

$\square$ lack of provider confidence

$\square$ lack of resources to address positive screen

$\square$ believe it is not indicated in our patient population

$\square$ inadequate evidence base to support screening $\square$ other

Yes

No a validated screening tool

an adaptation of a validated screening tool

an internally developed tool

unsure of original source

all visits

all well child visits

new patient visits only

when provider feels screening is indicated

$\square$ at specifically scheduled well child visits

$\square$ other 


\section{Confidential}

What method is used to screen for financial support?

Please describe other method.

Who introduces the screening process for family financial support? (choose all that apply)

Please note title of other person responsible for introducing the screening process.

When does the screening for family financial support occur?

Who is responsible for follow up of screening for family financial support? (choose all that apply)

Please note title of other person responsible for follow up.

Do you plan to begin screening for family financial support within the next 3 years?

Please describe the current plan for screening.

What barriers are currently in place that prohibit screening for family financial support? face-to-face interview

paper form

electronic questionnaire

other

preclinical staff

nurse/medical assistant

physician/ primary provider

social worker

$\square$ other

prior to the encounter date

on day of encounter at registration or in waiting room

on day of encounter during triage or rooming

during the encounter

preclinical staff

$\square$ nurse/medical assistant

$\square$ physician/ primary provider

$\square$ social worker

$\square$ other

Yes

No

lack of time

inadequate training

$\square$ lack of provider confidence

$\square$ lack of resources to address positive screen

$\square$ believe it is not indicated in our patient population

$\square$ inadequate evidence base to support screening other

Please describe other barriers to screening for family financial support.

Are patients/families at this continuity clinic site systematically screened for intimate partner violence?

(Example screening question: Have you ever been in a relationship in which you were physically hurt/threatened by a partner?)

What is the source of the screening questions on intimate partner violence?

a validated screening tool

an adaptation of a validated screening too

an internally developed tool

unsure of original source

Please name validated tool. 


\section{Confidential}

Page 7 of 22

At what types of visits is screening for intimate partner violence conducted? (choose all that apply)

Please describe other visit type.

What method is used to screen for intimate partner violence?

Please describe other method.

Who introduces the screening process for intimate partner violence? (choose all that apply)

preclinical staff

$\square$ nurse/medical assistant

physician/ primary provider

social worker

other

Please note title of other person responsible for introducing the screening process.

When does the screening for intimate partner violence occur?

Who is responsible for follow up of screening for intimate partner violence? (choose all that apply)

Please note title of other person responsible for follow up.

Do you plan to begin screening for intimate partner violence within the next 3 years?

$\bigcirc$ No

Please describe the current plan for screening.

What barriers are currently in place that prohibit screening for intimate partner violence?

lack of time

$\square$ inadequate training lack of provider confidence

lack of resources to address positive screen

believe it is not indicated in our patient population

$\square$ inadequate evidence base to support screening

other

Please describe other barriers to screening for intimate partner violence.

Are patients/families at this continuity clinic site systematically screened for maternal depression/family mental illness?

(Example screening question: Has anyone been sad or depressed at times?) 


\section{Confidential}

Page 8 of 22

What is the source of the screening questions on maternal depression/family mental illness?

Please name validated tool.

At what types of visits is screening for maternal depression/family mental illness conducted? (choose all that apply)

Please describe other visit type.

What method is used to screen for maternal depression/family mental illness?

Please describe other method.

Who introduces the screening process for maternal depression of family mental illness? (choose all that apply)

Please note title of other person responsible for introducing the screening process.

When does the screening for maternal depression/family mental illness occur?

Who is responsible for follow up of screening for maternal depression/family mental illness? (choose all that apply)

Please note title of other person responsible for follow up.

Do you plan to begin screening for maternal depression/family mental illness within the next 3 years?

Please describe the current plan for screening

What barriers are currently in place that prohibit screening for maternal depression/family mental illness? a validated screening tool

an adaptation of a validated screening tool

an internally developed tool

unsure of original source

all visits

all well child visits

$\square$ new patient visits only

$\square$ when provider feels screening is indicated

$\square$ at specifically scheduled well child visits

$\square$ other

e-to-face interview

paper form

electronic questionnaire

other

preclinical staff

nurse/medical assistant

physician/ primary provider

social worker

other

prior to the encounter date

on day of encounter at registration or in waiting room

on day of encounter during triage or rooming

during the encounter

preclinical staff

$\square$ nurse/medical assistant

$\square$ physician/ primary provider

social worker

$\square$ other

$\bigcirc$ Yes

No

lack of time

inadequate training

lack of provider confidence

lack of resources to address positive screen

$\square$ believe it is not indicated in our patient population

inadequate evidence base to support screening

other 
Please describe other barriers to screening for maternal depression/mental illness.

Are patients/families at this continuity clinic site systematically screened for household substance abuse?

(Example screening question: Do you or does anyone else in your home use drugs?)

What is the source of the screening questions on household substance abuse?

Please name validated tool.

At what types of visits is screening for household substance abuse conducted? (choose all that apply)

Please describe other visit type.

What method is used to screen for household substance abuse?

Please describe other method.

Who introduces the screening process for substance abuse? (choose all that apply)

Please note title of other person responsible for introducing the screening process.

When does the screening for household substance abuse occur?

Who is responsible for follow up of screening for household substance abuse? (choose all that apply)

Please note title of other person responsible for follow up.

Do you plan to begin screening for household substance abuse within the next 3 years?

Please describe the current plan for screening. $\bigcirc$ Yes

$\bigcirc$ No a validated screening tool

an adaptation of a validated screening too

an internally developed tool

unsure of original source

all visits

$\square$ all well child visits

$\square$ new patient visits only

$\square$ when provider feels screening is indicated

$\square$ at specifically scheduled well child visits

$\square$ other

face-to-face interview

paper form

electronic questionnaire

other

preclinical staff

nurse/medical assistant

physician/primary provider

social worker

$\square$ other

prior to the encounter date

on day of encounter at registration or in waiting room

on day of encounter during triage or rooming

during the encounter

preclinical staff

$\square$ nurse/medical assistant

$\square$ physician/ primary provider

$\square$ social worker

$\square$ other

$\bigcirc$ Yes

$\bigcirc$ No 


\section{Confidential}

Page 10 of 22

What barriers are currently in place that prohibit screening for household substance abuse?

Please describe other barriers to screening for household substance abuse.

Are patients/families at this continuity clinic site systematically screened for parental health literacy?

(Example screening question: How happy are you with how you read?)

What is the source of the screening questions on parental health literacy?

Please name validated tool.

At what types of visits is screening for parental health literacy conducted? (choose all that apply)

Please describe other visit type.

What method is used to screen for parental health literacy?

Please describe other method.

Who introduces the screening process for parental health literacy? (choose all that apply)

Please note title of other person responsible for introducing the screening process.

When does the screening for parental health literacy occur?

Who is responsible for follow up of screening for parental health literacy? (choose all that apply) lack of time

inadequate training

lack of provider confidence

$\square$ lack of resources to address positive screen

believe it is not indicated in our patient population

$\square$ inadequate evidence base to support screening other

$\bigcirc$ Yes

$\bigcirc$ No a validated screening tool

an adaptation of a validated screening too

an internally developed tool

unsure of original source

all visits

$\square$ all well child visits

new patient visits only

$\square$ when provider feels screening is indicated

at specifically scheduled well child visits

$\square$ other

face-to-face interview

paperform

electronic questionnaire

other

preclinical staff

nurse/medical assistant

physician/ primary provider

social worker

other

prior to the encounter date

on day of encounter at registration or in waiting room

on day of encounter during triage or rooming

during the encounter

$\square$ preclinical staff

$\square$ nurse/medical assistant

$\square$ physician/ primary provider

$\square$ social worker

$\square$ other 


\section{Confidential}

Please note title of other person responsible for follow up.

Do you plan to begin screening for parental health literacy within the next 3 years?

Please describe the current plan for screening.

What barriers are currently in place that prohibit screening for parental health literacy?

Please describe other barriers to screening for parental health literacy.

Are patients/families at this continuity clinic site systematically screened for parental stress?

(Example screening question: Do you wish you had more help with your child?)

What is the source of the screening questions on parental stress?

Please name validated tool.

At what types of visits is screening for parental stress conducted? (choose all that apply)

Please describe other visit type.

What method is used to screen for parental stress?

Please describe other method.

Who introduces the screening process for parental stress? (choose all that apply)

Please note title of other person responsible for introducing the screening process.

When does the screening for parental stress occur?
Yes

No

lack of time

$\square$ inadequate training

$\square$ lack of provider confidence

$\square$ lack of resources to address positive screen

believe it is not indicated in our patient population

$\square$ inadequate evidence base to support screening

$\square$ other

Yes

No

a validated screening too

an adaptation of a validated screening tool

an internally developed tool

unsure of original source

all visits

all well child visits

$\square$ new patient visits only

when provider feels screening is indicated

at specifically scheduled well child visits

other

face-to-face interview

paper form

electronic questionnaire

other

preclinical staff

$\square$ nurse/medical assistant

physician/ primary provider

$\square$ social worker

other

prior to the encounter date

on day of encounter at registration or in waiting room

on day of encounter during triage or rooming

during the encounter 
Who is responsible for follow up of screening for parental stress? (choose all that apply)

Please note title of other person responsible for follow up.

Do you plan to begin screening for parental stress within the next 3 years?

Please describe the current plan for screening.

What barriers are currently in place that prohibit screening for parental stress?

Please describe other barriers to screening for parental stress.

Are patients/families at this continuity clinic site systematically screened for childcare needs?

(Example screening question: Do you need daycare for your child? If YES, would you like help finding it?)

What is the source of the screening questions on childcare needs?

Please name validated tool.

At what types of visits is screening for childcare needs conducted? (choose all that apply)

Please describe other visit type.

What method is used to screen for childcare needs?

Please describe other method.

Who introduces the screening process for childcare needs? (choose all that apply)

Please note title of other person responsible for introducing the screening process. preclinical staff

nurse/medical assistant

$\square$ physician/ primary provider

social worker

$\square$ other

Yes

No

lack of time

inadequate training

$\square$ lack of provider confidence

$\square$ lack of resources to address positive screen

$\square$ believe it is not indicated in our patient population

$\square$ inadequate evidence base to support screening

$\square$ other

Yes

No

a validated screening tool

$\bigcirc$ an adaptation of a validated screening tool

an internally developed tool

unsure of original source

$\square$ all visits

all well child visits

$\square$ new patient visits only

$\square$ when provider feels screening is indicated

at specifically scheduled well child visits

other

face-to-face interview

paper form

electronic questionnaire

other

preclinical staff

nurse/medical assistant

physician/ primary provider

$\square$ social worker

$\square$ other 
When does the screening for childcare needs occur?

Who is responsible for follow up of screening for childcare needs? (choose all that apply)

Please note title of other person responsible for followup.

Do you plan to begin screening for childcare needs within the next 3 years?

Please describe the current plan for screening.

What barriers are currently in place that prohibit screening for childcare needs?

Please describe other barriers to screening for childcare needs.

Are patients/families at this continuity clinic site systematically screened for child learning problems/academic achievement?

(Example screening question: How is your child doing in school? Is he/she getting the help to learn what he/she needs?)

What is the source of the screening questions on child learning problems/academic achievement?

Please name validated tool.

At what types of visits is screening for child learning problems/academic achievement conducted? (choose all that apply)

Please describe other visit type.

What method is used to screen for child learning problems/academic achievement?

Please describe other method. prior to the encounter date

on day of encounter at registration or in waiting room

on day of encounter during triage or rooming

during the encounter

preclinical staff

nurse/medical assistant

$\square$ physician/ primary provider

social worker

$\square$ other

$\bigcirc$ Yes

$\bigcirc$ No

lack of time

inadequate training

lack of provider confidence

lack of resources to address positive screen

believe it is not indicated in our patient population

$\square$ inadequate evidence base to support screening

$\square$ other

Yes

$\bigcirc$ No a validated screening tool

an adaptation of a validated screening tool

an internally developed tool

unsure of original source

$\square$ all visits

all well child visits

$\square$ new patient visits only

$\square$ when provider feels screening is indicated

$\square$ at specifically scheduled well child visits

other

face-to-face interview

paper form

electronic questionnaire

other 
Who introduces the screening process for child problems/academic achievement? (choose all that apply)

Please note title of other person responsible for introducing the screening process.

When does the screening for child learning problems/academic achievement occur?

Who is responsible for follow up of screening for child learning problems/academic achievement? (choose all that apply)

Please note title of other person responsible for follow up.

Do you plan to begin screening for child learning problems/academic achievement within the next 3 years?

Please describe the current plan for screening.

What barriers are currently in place that prohibit screening for child learning problems/academic achievement?

Please describe other barriers to screening for child learning problems/academic achievement.

Are patients/families at this continuity clinic site systematically screened for problems with the home physical environment?

(Example screening question: In the last 12 months has the electric or gas company threatened to shut off the electricity or gas in your home?)

What is the source of the screening questions on home physical environment?

Please name validated tool.

At what types of visits is screening for home physical environment? (choose all that apply) preclinical staff learning

nurse/medical assistant

$\square$ physician/ primary provider

social worker

$\square$ other

prior to the encounter date

on day of encounter at registration or in waiting room

on day of encounter during triage or rooming

during the encounter

preclinical staff

$\square$ nurse/medical assistant

physician/ primary provider

social worker

$\square$ other

Yes

No

lack of time

$\square$ inadequate training

$\square$ lack of provider confidence

$\square$ lack of resources to address positive screen

believe it is not indicated in our patient population

inadequate evidence base to support screening

$\square$ other

Yes

No

a validated screening tool

an adaptation of a validated screening tool

an internally developed tool

unsure of original source

all visits

all well child visits

new patient visits only

$\square$ when provider feels screening is indicated

$\square$ at specifically scheduled well child visits

$\square$ other 
Please describe other visit type.

What method is used to screen for home physical environment?

Please describe other method.

Who introduces the screening process for home physical environment? (choose all that apply)

Please note title of other person responsible for introducing the screening process.

When does the screening for home physical environment occur?

Who is responsible for follow up of screening for home physical environment? (choose all that apply)

Please note title of other person responsible for follow up.

Do you plan to begin screening for problems with the home physical environment within the next 3 years?

Please describe the current plan for screening.

What barriers are currently in place that prohibit screening for home physical environment?

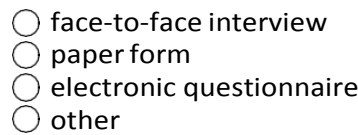

other

preclinical staff

$\square$ nurse/medical assistant

physician/ primary provider

social worker

other

prior to the encounter date

on day of encounter at registration or in waiting room

on day of encounter during triage or rooming

during the encounter

preclinical staff

$\square$ nurse/medical assistant

$\square$ physician/ primary provider

$\square$ social worker

other

$\bigcirc$ Yes

$\bigcirc$ No

lack of time

inadequate training

lack of provider confidence

lack of resources to address positive screen

believe it is not indicated in our patient

population

$\square$ inadequate evidence base to support screening

other

Please describe other barriers to screening for home physical environment.

Are patients/families at this continuity clinic site systematically screened for problems with the neighborhood physical environment?

(Example screening question: Are you worried about threats to your child's safety at school or in the neighborhood?)

What is the source of the screening questions on neighborhood environment? 


\section{Confidential}

Please name validated tool.

At what types of visits is screening for neighborhood physical environment conducted? (choose all that apply)

Please describe other visit type.

What method is used to screen for neighborhood physical environment?

Please describe other method.

Who introduces the screening process for neighborhood environment? (choose all that apply)

Please note title of the other person responsible for introducing the screening process.

When does the screening for neighborhood physical environment occur?

Who is responsible for follow up of screening for neighborhood physical environment? (choose all that apply)

Please note title of other person responsible for follow up.

Do you plan to begin screening for problems with the neighborhood physical environment within the next 3 years?

Please describe the current plan for screening.

What barriers are currently in place that prohibit screening for neighborhood physical environment? all visits all well child visits

new patient visits only

when provider feels screening is indicated

at specifically scheduled well child visits

$\square$ other

face-to-face interview

paper form

electronic questionnaire

other

preclinical staff physical

$\square$ nurse/medical assistant

physician/ primary provider

social worker

other

prior to the encounter date

on day of encounter at registration or in waiting room

on day of encounter during triage or rooming

during the encounter

preclinical staff

$\square$ nurse/medical assistant

physician/primary provider

$\square$ social worker

$\square$ other

$\bigcirc$ Yes

$\bigcirc$ No

lack of time

inadequate training

lack of provider confidence

$\square$ lack of resources to address positive screen

$\square$ believe it is not indicated in our patient population

$\square$ inadequate evidence base to support screening

$\square$ other

Please describe other barriers to screening for neighborhood physical environment. 
Are patients/families at this continuity clinic site systematically screened for food insecurity?

(Example screening question: In the last year, did you worry that your food would run out before you got money or food stamps to buy more?)

What is the source of the screening questions on food insecurity?

Please name validated tool.

At what types of visits is screening for food insecurity conducted? (choose all that apply)

Please describe other visit type.

What method is used to screen for food insecurity?

Please describe other method.

Who introduces the screening process for food insecurity? (choose all that apply)

Please note title of other person responsible for introducing the screening process.

When does the screening for food insecurity occur?

Who is responsible for follow up of screening for food insecurity? (choose all that apply)

Please note title of other person responsible for followup.

Do you plan to begin screening for food insecurity within the next 3 years?
Yes

No a validated screening tool

an adaptation of a validated screening tool

an internally developed tool

unsure of original source

all visits

all well child visits

$\square$ new patient visits only

when provider feels screening is indicated

at specifically scheduled well child visits

other

face-to-face interview

paper form

electronic questionnaire

other

preclinical staff

nurse/medical assistant

physician/primary provider

$\square$ social worker

other

prior to the encounter date

on day of encounter at registration or in waiting room

on day of encounter during triage or rooming

during the encounter

preclinical staff

$\square$ nurse/medical assistant

$\square$ physician/ primary provider

$\square$ social worker

$\square$ other

Yes

No

Please describe the current plan for screening. 


\section{Confidential}

Page 18 of 22

What barriers are currently in place that prohibit screening for food insecurity?

Please describe other barriers to screening for food insecurity.

Are patients/families at this continuity clinic site systematically screened for parental incarceration?

(Example screening question:Has anyone been in jail/is anyone now in jail?)

What is the source of the screening questions on parental incarceration?

Please name validated tool.

At what types of visits is screening for parental incarceration conducted? (choose all that apply)

Please describe other visit type.

What method is used to screen for parental incarceration?

Please describe other method.

Who introduces the screening process for parental incarceration? (choose all that apply)

Please note title of other person responsible for introducing the screening process.

When does the screening for parental incarceration occur?

Who is responsible for follow up of screening for parental incarceration? (choose all that apply) lack of time

$\square$ inadequate training

lack of provider confidence

lack of resources to address positive screen

believe it is not indicated in our patient population

inadequate evidence base to support screening other

Yes

No

a validated screening too

an adaptation of a validated screening too

an internally developed tool

unsure of original source

all visits

all well child visits

$\square$ new patient visits only

when provider feels screening is indicated

$\square$ at specifically scheduled well child visits

other

face-to-face interview

paper form

electronic questionnaire

other

preclinical staff

nurse/medical assistant

physician/ primary provider

$\square$ social worker

other

prior to the encounter date

on day of encounter at registration or in waiting room

on day of encounter during triage or rooming

during the encounter

$\square$ preclinical staff

$\square$ nurse/medical assistant

$\square$ physician/primary provider

social worker

$\square$ other 
Please note title of other person responsible for follow up.

Do you plan to begin screening for parental incarceration within the next 3 years?

Yes

No

Please describe the current plan for screening.

What barriers are currently in place that prohibit screening for parental incarceration?

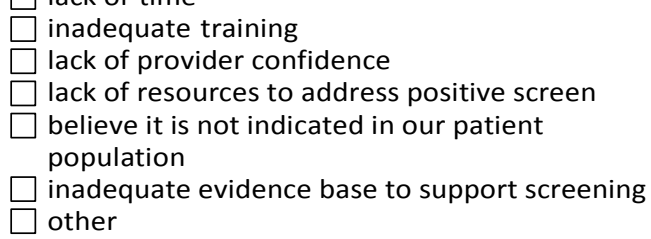

Please describe other barriers to screening for parental incarceration.

Are patients/families at this continuity clinic site systematically screened for immigration status?

Yes

No

(Example screening question: Do you have any questions about your family's immigration status? Do you need help accessing benefits or services for your family?)

What is the source of the screening questions on immigration status?

Please name validated tool.

At what types of visits is screening for immigration status conducted? (choose all that apply)

all visits

all well child visits

new patient visits only

when provider feels screening is indicated

$\square$ at specifically scheduled well child visits

other

Please describe other visit type.

What method is used to screen for immigration status?

face-to-face interview
paper form
electronic questionnaire
other

Please describe other method.

Who introduces the screening process for immigration status? (choose all that apply)

preclinical staff

nurse/medical assistant

$\square$ physician/ primary provider

social worker

$\square$ other

Please note title of other person responsible for introducing the screening process. 
When does the screening for immigration status occur?

Who is responsible for follow up of screening for immigration status? (choose all that apply)

Please note title of other person responsible for followup.

Do you plan to begin screening for immigration status within the next 3 years?

Please describe the current plan for screening.

What barriers are currently in place that prohibit screening for immigration status?

Please describe other barriers to screening for immigration status.

Are patients/families at this continuity clinic site systematically screened for firearm exposure?

(Example screening question: Is there a gun in your home?)

What is the source of the screening questions on firearm exposure?

Please name validated tool.

At what types of visits is screening for firearm exposure conducted? (choose all that apply)

Please describe other visit type.

What method is used to screen for firearm exposure? prior to the encounter date

on day of encounter at registration or in waiting room

on day of encounter during triage or rooming

during the encounter

$\square$ preclinical staff

nurse/medical assistant

$\square$ physician/ primary provider

$\square$ social worker

$\square$ other

Yes

No

lack of time

$\square$ inadequate training

$\square$ lack of provider confidence

$\square$ lack of resources to address positive screen

$\square$ believe it is not indicated in our patient population

$\square$ inadequate evidence base to support screening

$\square$ other

Yes

No

a validated screening too

an adaptation of a validated screening tool

an internally developed tool

unsure of original source

$\square$ all visits

$\square$ all well child visits

$\square$ new patient visits only

$\square$ when provider feels screening is indicated

$\square$ at specifically scheduled well child visits $\square$ other

\footnotetext{
face-to-face interview

paper form

electronic questionnaire

other
}

Please describe other method. 
exposure? (choose all that apply)

Please note title of other person responsible for introducing the screening process.

When does the screening for firearm exposure occur?

Who is responsible for follow up of screening for firearm exposure? (choose all that apply)

Please note title of other person responsible for follow up.

Do you plan to begin screening for firearm exposure within the next 3 years?

Please describe the current plan for screening.

What barriers are currently in place that prohibit screening for firearm exposure? nurse/medical assistant

physician/ primary provider

social worker

other prior to the encounter date

on day of encounter at registration or in waiting room

on day of encounter during triage or rooming

during the encounter

preclinical staff

nurse/medical assistant

physician/ primary provider

social worker

other

Yes

No lack of time

inadequate training

$\square$ lack of provider confidence

$\square$ lack of resources to address positive screen

$\square$ believe it is not indicated in our patient population

$\square$ inadequate evidence base to support screening $\square$ other

Please describe other barriers to screening for firearm exposure.

We are also curious about current practices for identifying resilience in your clinic. Resilience is the ability of people to withstand, adapt to, and recover from adversity and stress.

Are the patients/families at your continuity clinic site systematically screened for any indicators of resilience?

What is the source of the screening questions on resilience?

Please name validated tool. $\bigcirc$ Yes
No

a validated screening tool

an adaptation of a validated screening tool

an internally developed tool

unsure of original source 


\section{Confidential}

Page 22 of 22

Please select the resilience factors for which you are screening. (choose all that apply)

Please name any other resilience factors for which you are screening.

Please provide any additional comments about screening for social determinants of health and/or resilience in your clinic: $\square$ family/social support

positive outlook

spirituality

$\square$ adaptability to change

family communication

financial management

$\square$ family time/shared recreation/routines and rituals

$\square$ parental confidence

$\square$ other 


\section{APPENDIX B: Limited Systematic Review}

A Systematic Review of Interventions to Address Food Insecurity in Children

Marcia Morgenlander, MD

marcia.morgenlander@unchealth.unc.edu

Word Count: 1619 


\section{Rationale}

Food insecurity affects nearly 16 million US households ${ }^{1}$. Children living in 3 million of those households do not have access to adequate nutritious food. ${ }^{1}$ In addition to the moral repugnance of children experiencing hunger, significant public health consequences follow as the wages of early food insecurity manifest over the life course.

Undernourished infants and toddlers may display deficits in growth and neurocognitive development. ${ }^{2}$ Children from food insecure households are at risk for poor school performance. ${ }^{3}$ They may suffer from poor growth due to food restriction. ${ }^{4,5}$ Alternatively, as they reach school age, these children may be more prone to obesity resulting from unhealthy food choices. ${ }^{6,7} \mathrm{High}$ rates of food insecurity are found in children with chronic conditions such as asthma ${ }^{8}$ and Type 2 diabetes. ${ }^{9}$ Neurodevelopmental consequences of food insecurity include externalizing behaviors, anxiety, depression, inattention and poor academic performance which persist into adolescence and adulthood. ${ }^{2}$ Furthermore, poor nutrition in childhood is associated with increased incidence of diabetes and cardiovascular disease in adulthood. ${ }^{10}$

There is agreement in the literature that food insecurity contributes to morbidity in childhood and beyond, but there is less consensus on appropriate methods for health care providers to address it. The American Academy of Pediatrics (AAP) recommends routine screening for household food insecurity in outpatient primary care settings. ${ }^{10}$ Several validated screening tools include a commonly used 2 question screen. ${ }^{11}$ In addition to screening, the AAP statement encourages 
pediatricians to familiarize themselves with resources such as WIC, SNAP and local food pantries, to link patients to these resources, and to provide follow-up. ${ }^{10}$

Educational and quality improvement initiatives can enhance provider knowledge, and increase rates of screening and detection of food insecurity in outpatient settings. ${ }^{12-14}$ Still, although pediatric providers acknowledge the importance of food insecurity and express willingness to screen, only about $15 \%$ actually do so. ${ }^{15}$ In a busy practice, possible provider barriers to screening for food insecurity include lack of knowledge about or comfort with the subject, lack of time, inadequate personnel or infrastructure, or concern that patients may be stigmatized. The barrier to FI screening most cited by providers is not knowing how to handle a positive screen. ${ }^{15}$ The lack of clarity on interventions for food insecurity is the impetus for this systematic review.

\section{Objectives}

The first objective of this limited systematic review is to compile evidence to answer the question, "Do primary care feasible or referable interventions to address food insecurity in children result in increased referrals of families to services, increased use of services by families, improved family physical or emotional well-being, or improved child health outcomes?"

The second objective is to ask "What are the harms of primary care-feasible or referable interventions to address food insecurity?"

\section{Methods}

The protocol for this limited systematic review was not formally registered in PROSPERO.

The author's search strategy was determined by the intention to capture articles describing and evaluating interventions for food insecurity in children that were initiated in a primary care 
setting rather than in community-based, hospital or subspecialty settings. The first search algorithm used the terms "food insecurity" AND "pediatrics" AND "primary care." Because food insecurity is one of the "social determinants of health" the author then included this term in the search to return articles that screened and intervened for food insecurity in combination with other social determinants such as poverty or inadequate housing.

The final search of PubMed and Cochrane databases took place on April 6, 2017. The final algorithms used these operators and MeSH terms:

(pediatric [tw] OR pediatrics [tw] OR pediatrician[tw] OR pediatrics[MeSH]) AND ("food insecurity"[tw] OR (food AND "social determinants of health")) in PubMed

(pediatric OR pediatrics OR pediatrician) AND ("food insecurity" OR (food AND "social determinants of health")) in Cochrane. This search is filtered for human subjects and English language. Clinicaltrials.gov was also searched for "food insecurity" and revealed no pertinent unpublished or gray literature. One study title in clinicaltrials.gov may be pertinent to a future systematic review, however, the study had not yet begun recruitment. Abstracts from the resulting records were reviewed for inclusion in the systematic review by the author using the following criteria:

\begin{tabular}{|l|l|l|}
\hline PICOTS & Include & Exclude \\
\hline Population & $\begin{array}{l}\text { Primary care pediatric patients birth through } \\
18 \text { years of age and/or their families }\end{array}$ & $\begin{array}{l}\text { Adults over 18 years of age. } \\
\text { Pediatric populations drawn } \\
\text { from subspecialty clinics, } \\
\text { hospitals or emergency } \\
\text { departments }\end{array}$ \\
\hline Intervention & $\begin{array}{l}\text { Services to address food insecurity that may } \\
\text { result from a referral by the primary care } \\
\text { provider. These services may be implemented } \\
\text { by non-clinicians and may be aimed at the } \\
\text { caregiver, child or family }\end{array}$ & $\begin{array}{l}\text { Community based } \\
\text { interventions. Interventions } \\
\text { directed toward obesity. } \\
\text { Interventions directed toward } \\
\text { housing, transportation, }\end{array}$ \\
\hline
\end{tabular}




\begin{tabular}{|c|c|c|}
\hline & & $\begin{array}{l}\text { family mental health or } \\
\text { substance abuse or child } \\
\text { physical or sexual abuse }\end{array}$ \\
\hline Comparison & $\begin{array}{l}\text { Intervention group compared to usual care or } \\
\text { a combined intervention which allows } \\
\text { assessment of the contribution of the primary } \\
\text { intervention. } \\
\text { Pre and post assessments }\end{array}$ & $\begin{array}{l}\text { No comparison. Combined } \\
\text { intervention which does not } \\
\text { allow assessment of the } \\
\text { primary intervention }\end{array}$ \\
\hline Outcomes & $\begin{array}{ll}\text { - Increased referrals of families to } \\
\text { services } \\
\text { - } \\
\text { Increased utilization of services by } \\
\text { families } \\
\text { Improved family physical, social or } \\
\text { emotional well-being including } \\
\text { enhanced patient-provider relationship } \\
\text { (include validated scales or self-report) } \\
\text { - Improved child health outcomes (may } \\
\text { include improvement in growth } \\
\text { parameters, increased compliance } \\
\text { with scheduled WCC visits, increased } \\
\text { immunization rates, decreased school } \\
\text { absenteeism, decreased ED visits, or } \\
\text { other objective measures of improved } \\
\text { child health }\end{array}$ & $\begin{array}{l}\text { Outcomes not otherwise } \\
\text { specified }\end{array}$ \\
\hline Timing & No limits specified & \\
\hline Setting & $\begin{array}{l}\text { Studies conducted in countries categorized as } \\
\text { very high on the Human Development Index } \\
\text { as defined by the United Nations } \\
\text { Development Program }\end{array}$ & $\begin{array}{l}\text { Studies conducted in } \\
\text { countries less than very high } \\
\text { on the Human Development } \\
\text { Index. }\end{array}$ \\
\hline $\begin{array}{l}\text { Study } \\
\text { Designs }\end{array}$ & $\begin{array}{l}\text { RCTs, systematic reviews, cohort trials with a } \\
\text { control group, case-control studies, pre and } \\
\text { post assessments }\end{array}$ & $\begin{array}{l}\text { Case studies, narrative } \\
\text { reviews, editorials, } \\
\text { commentaries. }\end{array}$ \\
\hline
\end{tabular}

Included articles were hand-searched for other pertinent articles, after which data were abstracted from included articles to compare associations with interventions for food insecurity and measured outcomes. Risk of bias of individual studies and across studies was assessed. Overall outcome trends for interventions were combined and analyzed when possible. 


\section{Results}

The PubMed search returned 63 articles. The search of Cochrane returned 7 articles, for a total of 70 articles. After duplicates were removed, 64 articles remained. Hand search did not return any additional articles. 15 articles were removed because of ineligible population. 37 were ineligible because of the lack of appropriate intervention. Six were removed because they were narratives rather than experimental study designs. Six publications remained for inclusion in qualitative analysis. Due to the paucity of available literature, original PICOTS were liberalized to include pilot implementations even in the absence of a comparison group. PRISMA diagram is below.

FIGURE 1: PRISMA DIAGRAM

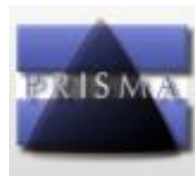

PRISMA DIAGRAM

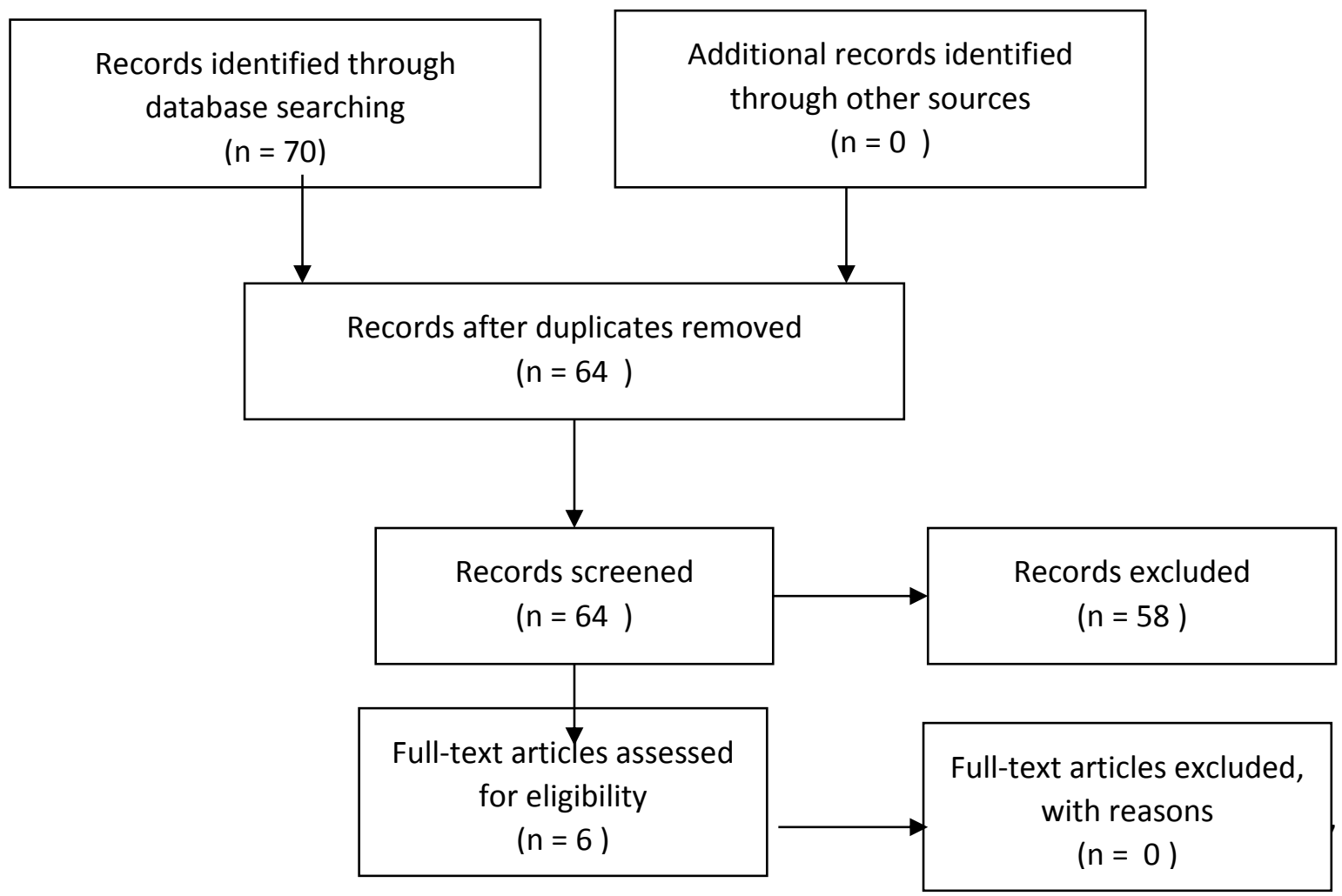


Studies included in

qualitative synthesis

$(n=6)$

All six of the included publications assessed the implementation of screening for food insecurity and referral to some type of service. Three studies used the two question food insecurity screen, two used the six item screen, and one study used the comprehensive Parent Screening Questionnaire (PSQ) which includes the domain of food insecurity in a questionnaire which addresses broader social needs. Only one study, Feigelman, was a randomized control trial (RCT), and in that trial, the residents were randomized to an educational program on screening, or none prior to implementation. The age of the targeted patient populations and the outcomes are heterogeneous. See Table 2 for further detail.

\section{Table 2 Study Characteristics}

\begin{tabular}{|l|l|l|l|}
\hline Publication Author & Population & Intervention & Outcomes \\
\hline Smith et al. & $\begin{array}{l}\text { Caregivers of all } \\
\text { patients of student } \\
\text { run free pediatric } \\
\text { clinics }\end{array}$ & $\begin{array}{l}\text { Paper 6 item screen } \\
\text { and referral }\end{array}$ & $\begin{array}{l}\text { Increased } \\
\text { identification of FI } \\
\text { Increased linkage to } \\
\text { community food } \\
\text { resources } \\
\text { Increased SNAP } \\
\text { enrollment }\end{array}$ \\
\hline Bottino et al. & $\begin{array}{l}\text { Caregivers of 3-10 } \\
\text { year old patients }\end{array}$ & $\begin{array}{l}\text { Electronic 6 item } \\
\text { screen and referral } \\
\text { menu }\end{array}$ & $\begin{array}{l}\text { Incomplete overlap } \\
\text { between food } \\
\text { insecurity and desire } \\
\text { for referrals }\end{array}$ \\
\hline Adams et al. & $\begin{array}{l}\text { Caregivers in } \\
\text { academic pediatric } \\
\text { resident clinic }\end{array}$ & $\begin{array}{l}\text { Paper 2 question } \\
\text { screen and provision } \\
\text { of electronic } \\
\text { resources }\end{array}$ & $\begin{array}{l}\text { Acceptability to } \\
\text { providers }\end{array}$ \\
\hline
\end{tabular}




\begin{tabular}{|c|c|c|c|}
\hline & & $\begin{array}{l}\text { (Screen and } \\
\text { Intervene) }\end{array}$ & \\
\hline Beck et al. & $\begin{array}{l}\text { Food insecure } \\
\text { families with infants } \\
<12 \text { months }\end{array}$ & $\begin{array}{l}\text { Paper } 2 \text { question } \\
\text { screen } \\
\text { Provision of infant } \\
\text { formula for FI or at } \\
\text { provider discretion } \\
\text { (KIND) }\end{array}$ & $\begin{array}{l}\text { Improved preventive } \\
\text { services } \\
\text { No difference in } \\
\text { weight for length at } \\
\text { age } 9 \text { months }\end{array}$ \\
\hline Fox et al. & $\begin{array}{l}\text { Caregivers of all new } \\
\text { patients to pediatric } \\
\text { weight management } \\
\text { clinic }\end{array}$ & $\begin{array}{l}\text { Paper } 2 \text { question } \\
\text { screen and referral to } \\
\text { SNAP if eligible }\end{array}$ & $\begin{array}{l}\text { Only } 8 \% \text { of those } \\
\text { referred to SNAP } \\
\text { enrolled }\end{array}$ \\
\hline Feigelman et al. & $\begin{array}{l}\text { Residents and } \\
\text { caregivers of } 0-5 \text { year } \\
\text { old patients }\end{array}$ & $\begin{array}{l}\text { Resident education } \\
\text { Administration of } \\
\text { Parent Screening } \\
\text { Questionnaire } \\
\text { (SEEK) }\end{array}$ & $\begin{array}{l}\text { Residents expressed } \\
\text { increased comfort } \\
\text { with screening. } \\
\text { Parents report } \\
\text { increased satisfaction } \\
\text { with doctor/patient } \\
\text { relationship. }\end{array}$ \\
\hline
\end{tabular}

The risk of bias for each of the six studies was assessed using the critical appraisal template from the PUBH 752 Seminar in Critical Appraisal of Health Literature at the UNC Gillings School of Global Public Health. All of the six included studies were deemed to have a high risk of bias. In five of the studies high risk of bias was based on no randomization, no comparison group, and no blinding of patients or investigators. In the one RCT, randomization was day of the week of resident continuity clinic. Residents self-reported increased comfort and likelihood of screening, however, residents were aware of the goals of the study and the desirability of affirmative responses to screening.

In answer to our first key question, "Do primary care feasible or referable interventions to address food insecurity in children result in increased referrals of families to services, increased utilization of services by families, improved family physical or emotional well-being, or 
improved child health outcomes?" four of the six studies implemented a screening and referral process that, by definition, increased referrals compared to no referrals for food insecurity being made prior to program implementation. No study actually compared provider referrals made or enrollment in WIC or SNAP between groups who were and were not screened for food insecurity. The one study that followed up referrals, showed that only $8 \%$ of those referred actually completed a referral. The Keeping Infants Nourished and Developing (KIND) intervention by Beck et al. provided a direct linkage embedded in the clinic so that all eligible families actually received formula. KIND families who received formula were compared with families not receiving KIND. KIND families were more likely to have successfully completed recommended well child encounters and more likely to have had lead and developmental screening. Two studies indicated that food insecurity screening is acceptable to providers and one showed that education increases provider comfort level of screening. In the one study that measured acceptability to caregivers, they reported improved doctor patient relationship in clinics screening for food insecurity. Only the KIND study measured a health outcome, weight for length at age 9 months. There was no difference between intervention and comparison group. However, this is probably a desirable outcome since infants receiving formula through the intervention might be expected to be smaller, so that equivalent growth may indicate successful intervention.

Our second question cannot be addressed in this systematic review since none of the six included studies mentioned harms of screening and intervention.

The heterogeneity of measured outcomes is unsuitable for meta-analysis. The risk of bias across studies is high since there are not consistently or reproducibly demonstrated outcomes. 


\section{Discussion}

Overall this review suggests that interventions can increase the level of screening, increase the identification of food insecurity, and increase the number of referrals made. Referrals may not actually lead to linkage to services, and patients who desire referrals may be missed by screening. Patients and providers are both amenable to this screening.

The strength of evidence for these conclusions is weak due to the low number of studies, the lack of experimental study designs and the heterogeneity of outcome measures.

Many questions remain regarding the effectiveness and efficacy of interventions for food insecurity. Ethical considerations prohibit detecting food insecurity and not offering interventions. But once identified as food insecure, families could be randomized to various interventions. We still need to learn whether making a referral or providing a resource leads to actual linkage to services. If the linkage occurs, is it sustainable, and does it actually lead to improved health outcomes? The existing evidence does not yet provide these answers.

The major limitations of this systematic review are the low number and the low quality of studies that evaluate primary care interventions for food insecurity.

\section{Conclusions}

Pediatricians have unique access to families of young children and seek ways to improve child health. The AAP recommends screening for food insecurity and making appropriate referrals. This initial review suggests that pediatric medical homes can successfully identify food insecure patients, create resource guides and forge community partnerships to address food insecurity. At this point, the evidence is insufficient to show benefits of primary care interventions for food 
insecurity. Studies of specific interventions and clearly defined outcome measures, including child health outcomes, are needed to inform future practice.

\section{References}

1. Coleman-Jennings A RM, Gregory C, Singh A. Household Food Insecurity in the United States in 2015. USDA Economic Research Report. 2016;215.

2. Shankar P, Chung R, Frank DA. Association of Food Insecurity with Children's Behavioral, Emotional, and Academic Outcomes: A Systematic Review. J Dev Behav Pediatr. 2017;38(2):135-150.

3. Predictors of poor school readiness in children without developmental delay at age 2 . Pediatrics 138 (2) (no pagination), 2016 Article Number: e20154477 Date of Publication: August 2016. 2016. http://onlinelibrary.wiley.com/o/cochrane/clcentral/articles/390/CN01196390/frame.html.

4. Conlon B, McGinn A, Lounsbury D, et al. The Role of Parenting Practices in the Home Environment among Underserved Youth. Childhood obesity (Print). 2016;11(4):394-405. http://onlinelibrary.wiley.com/o/cochrane/clcentral/articles/398/CN01170398/frame.html.

5. Bauer KW, MacLehose R, Loth KA, Fisher JO, Larson NI, Neumark-Sztainer D. Eatingand weight-related parenting of adolescents in the context of food insecurity. J Acad Nutr Diet. 2015;115(9):1408-1416.

6. Bhawra J, Cooke MJ, Hanning R, Wilk P, Gonneville SL. Community perspectives on food insecurity and obesity: Focus groups with caregivers of metis and Off-reserve first nations children. International journal for equity in health. 2015;14:96.

7. Nackers LM, Appelhans BM. Food insecurity is linked to a food environment promoting obesity in households with children. J Nutr Educ Behav. 2013;45(6):780-784.

8. Hendrickson MA, O'Riordan MA, Arpilleda JC, Heneghan AM. Effects of food insecurity on asthma outcomes in the pediatric emergency department. Pediatr Emerg Care. 2010;26(11):823-829.

9. Heerman WJ, Wallston KA, Osborn CY, et al. Food insecurity is associated with diabetes self-care behaviours and glycaemic control. Diabet Med. 2016;33(6):844-850.

10. Promoting Food Security for All Children. Pediatrics. 2015;136(5):e1431-1438. 
11. Hager ER, Quigg AM, Black MM, et al. Development and validity of a 2-item screen to identify families at risk for food insecurity. Pediatrics. 2010;126(1):e26-32.

12. Colvin JD, Bettenhausen JL, Anderson-Carpenter KD, Collie-Akers V, Chung PJ. Caregiver Opinion of In-Hospital Screening for Unmet Social Needs by Pediatric Residents. Acad Pediatr. 2016;16(2):161-167.

13. Colvin JD, Bettenhausen JL, Anderson-Carpenter KD, et al. Multiple Behavior Change Intervention to Improve Detection of Unmet Social Needs and Resulting Resource Referrals. Acad Pediatr. 2016;16(2):168-174.

14. Burkhardt MC, Beck AF, Conway PH, Kahn RS, Klein MD. Enhancing accurate identification of food insecurity using quality-improvement techniques. Pediatrics. 2012;129(2):e504-510.

15. Barnidge E, LaBarge G, Krupsky K, Arthur J. Screening for Food Insecurity in Pediatric Clinical Settings: Opportunities and Barriers. J Community Health. 2017;42(1):51-57. 